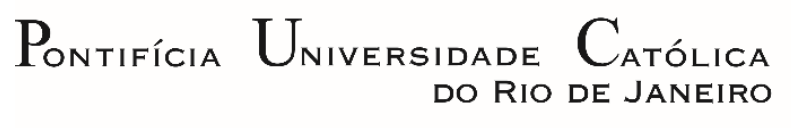

DO RIO DE JANEIRO

Felipe Zacharias de Sá

\title{
Análise Estrutural do Vaso de Pressão VP-CTVP-E-01
}

Projeto de Graduação

Projeto de Graduação apresentado ao Departamento de Engenharia Mecânica da PUC-Rio

Orientador: Professor José Luiz de França Freire Co-Orientador: Professor José Eduardo Maneschy

Rio de Janeiro

Dezembro de 2016 


\section{Agradecimentos}

Primeiramente, agradeço a Deus, pois sem ele eu não teria conseguido.

Quero agradecer ao meu orientador, Prof. José Luiz de França Freire, por suas valiosas orientações e importantes esclarecimentos, assim como pela sua disponibilidade em tirar todas as dúvidas que surgiram ao longo do trabalho.

Também agradeço ao meu co-orientador, Prof. José Eduardo Maneschy, por suas orientações, por ter se reunido comigo diversas vezes, pelas dicas valiosas que me deu e por ter revisado meu trabalho continuamente, corrigindo erros e sugerindo melhoras.

Finalmente agradeço ao Departamento de Engenharia Mecânica da PUC-Rio, junto com seus professores e coordenadores e ao meu pai, por todo o apoio que sempre me deu. 


\section{Resumo}

\section{Análise Estrutural do Vaso de Pressão VP-CTVP-E-01}

Vasos de pressão são usados para armazenar fluidos sob um diferencial de pressão interna-externa ao vaso de mais de uma atmosfera. Geralmente são projetados para resistir a pressões internas, mas também podem ser projetados para resistir a pressões externas. Podem estar sujeitos a variações de pressão e temperatura, além de outras formas de carregamentos externos. São usados para uma ampla gama de aplicações, desde armazenar líquidos sob altas pressões (e.g., centrais nucleares), até vasos que tenham que resistir a pressões externas (e.g., submarinos).

Vasos de pressão possuem diferentes geometrias e tamanhos, e podem ser classificados como sendo de paredes finas ou grossas. Os principais tipos são os cilíndricos (horizontal ou vertical) e os esféricos, mas podem assumir outras geometrias, assim como ter seções com geometrias diferentes (como o VP-CTVP-E-01).

Neste trabalho, é analisado um vaso de pressão que já foi projetado, o VP-CTVP-E-01. Primeiro é feita uma análise estrutural estática, através do cálculo e comparação da máxima pressão admissível de trabalho pela Divisão 1 e 2 da norma ASME, além da determinação da pressão de teste hidrostático (PTH). Em seguida, é feita uma análise completa de fadiga em toda a estrutura, tanto longe das descontinuidades como nos pontos críticos, usando a teoria proposta pelo Método SN. Para tanto, é assumido que o vaso será submetido a ciclos de variações de pressão que induzirão tensões alternadas cíclicas e elásticas ao longo da estrutura. No final, é estimada a vida do vaso.

Palavras chave: Vaso de pressão; análise estrutural; fadiga; ASME. 


\section{Abstract}

\section{Structural Analysis of the Pressure Vessel VP-CTVP-E-01}

Pressure vessels are used to store fluids under an internal-external pressure differential to the vessel of more than one atmosphere. They are usually designed to withstand internal pressures, but can also be designed to withstand external pressures. They may be subject to pressure and temperature variations, as well as other forms of external loadings. They are used for a wide range of applications, from storing liquids under high pressures (e.g., nuclear power plants) to vessels having to withstand external pressures (e.g., submarine).

Pressure vessels have different geometries and sizes, and can be classified as thin-walled or thick-walled. The main types are cylindrical (horizontal or vertical) and spherical, but they may assume other geometries. They may as well have sections with different geometries (such as the VP-CTVP-E-01).

First, a static structural analysis is performed, by calculating and comparing the maximum allowable working pressure by Division 1 and 2 of the ASME Boiler and Pressure Vessel Code. In addition it is determined the hydrostatic test pressure (HTP). Then, a complete fatigue analysis is done throughout the structure, both away from discontinuities and at critical points. It is used the theory proposed by the SN Method for that. In order to do so, it is assumed that the vessel will be subjected to cycles of pressure variations. These cycles will induce alternating cyclic and elastic stresses throughout the structure. In the end, the life of the vessel is estimated.

Key words: Pressure vessel; structural analysis; fatigue; ASME Boyler and Pressure Vessel Code. 


\section{SUMÁRIO}

1 INTRODUÇÃO___

1.1 O vaso VP-CTVP-E-01__ 7

1.2 Objetivos__ 11

1.3 Material didático utilizado___ 11

1.4 Divisão 1 x Divisão 2

2 MATERIAL___ 13

3 ANÁLISE ESTRUTURAL ESTÁTICA___ 15

3.1 Corrosão___ 15

3.2 Cálculo da MPAT pela Divisão $1 \_16$

3.3 Cálculo da PTH__ 20

3.4 Cálculo da MPAT pela Divisão $2 \ldots 21$

3.5 Comparação dos resultados___ 28

4 ANÁLISE dA FADIGA NO VASO DE PRESSÃO_ 29

4.1 Avaliação da fadiga segundo a metodologia $\mathrm{SN}_{2} \quad 30$

4.1.1 Quantificando a resistência à fadiga da estrutura___ 31

4.1.2 Análise das tensões atuantes___ 34 34

4.1.3 Acúmulo do dano à fadiga___ 39

4.1.4 Análise do dano à fadiga nos pontos críticos___ 42

4.2 Avaliação da fadiga segundo a norma ASME___ 52

5 CONCLUSÃO E SUGESTÕES PARA TRABALHOS FUTUROS 54

REFERÊNCIAS BIBLIOGRÁFICAS 55

ANEXO A - CÁLCULO DA CAPACIDADE VOLUMÉTRICA 56 ANEXO B - TENSÕES PRINCIPAIS EM VASOS CILÍNDRICOS 56 


\section{Lista de Figuras}

1.1 Desenho técnico do vaso VP-CTVP-E-01, parte $1 \quad 8$

1.2 Desenho técnico do vaso VP-CTVP-E-01, parte 29

1.3 Numeração das juntas soldadas 10

3.1 Casco cilíndrico (3D) 16

3.2 Tampo torisférico (3D) 17

3.3 Casco cônico (3D) 18

3.4 Tampo torisférico (2D) 22

3.5 Tampo elipsoidal (2D) 22

3.6 Seção cônica (2D) 26

4.1 Principais variáveis dimensionais do vaso de pessão [7] 34

4.2 Pontos críticos do vaso de pressão [7] 43

B-1 Tanque AB e as suas seções (a), (b) e (c) [13] 57 


\section{Lista de Tabelas}

$\begin{array}{lll}1.1 & \text { Eficiências das juntas soldadas } & 10\end{array}$

2.1 Propriedades mecânicas do SA-516 Gr 60 na temperatura ambiente

2.2 Propriedades mecânicas do SA-516 Gr 60 a $190{ }^{\circ} \mathrm{C}$

3.1 Resultados das MPAT segundo a Divisão 1 19

3.2 Resultados das MPAT para a temperatura ambiente segundo

a Divisão 2

3.3 Resultados das MPAT para $190{ }^{\circ} \mathrm{C}$ segundo a Divisão $2 \quad 27$

3.4 Aumento percentual da MPAT da Divisão 1 para

Divisão 2, temperatura ambiente 28

3.5 Aumento percentual da MPAT da Divisão 1 para

Divisão 2, temperatura de operação

4.1 Vidas à fadiga obtidas nas seções do vaso

4.2 Tensões longitudinais e circunferenciais dos 14 possíveis pontos críticos [7] 44

4.3 Constantes das curvas SN normalizadas pelo IIW [12] 45

4.4 Classes de soldagem normalizadas pelo IIW [12] 45

4.5 Vidas à fadiga nos pontos críticos 51 


\section{INTRODUÇÃO}

Vasos de pressão e tanques de armazenamento são reservatórios cuja função é armazenar fluidos. Os fluidos podem ser de estado gasoso ou líquido. Quando a diferença entre a pressão interna e externa ao vaso é inferior a uma atmosfera (ou 0,1 MPa), o reservatório é classificado como tanque de armazenamento. Quando esta diferença de pressões é superior a 0,1 MPa, o reservatório é classificado como vaso de pressão.

Muitas vezes, vasos de pressão podem estar submetidos a condições severas, sob altas pressões e altas temperaturas. Podem ainda armazenar fluidos inflamáveis ou radioativos, onde um eventual vazamento seria catastrófico. Desta forma, visando garantir a integridade do vaso e segurança, vasos de pressão devem seguir normas já aprovadas por muitos anos de uso. No Brasil, uma das normas seguida é a ASME (American Society of Mechanical Engineers).

\section{1}

\section{O vaso VP-CTVP-E-01}

O vaso de pressão deste trabalho é um vaso escola, cujo projeto foi encomendado à Petrobras pelo DEM da PUC-Rio. Ele é cilíndrico e tem um tampo elipsoidal. Sua outra extremidade é formada por um tronco cônico, que é soldado ao cilindro em sua base maior e a um bocal de visita em sua base menor. O costado é biapoiado (apoios do tipo sela). Há um bocal de entrada para o fluido, um dreno e uma torre de ventilação. O corpo principal é constituído de chapas calandradas unidas por soldagem. Todas as demais partes são também fixadas por solda. É considerado que o metal da base e o metal da solda são feitos do mesmo material, aço carbono ASTM A516 Gr. 60.

O fluido armazenado é a água, que varia da temperatura ambiente (quando o vaso estiver fora de operação) até $190{ }^{\circ} \mathrm{C}$ (quando o vaso estiver 
em operação). A água está sempre em estado líquido. Desta forma, a pressão interna será sempre maior que a pressão de saturação para dada temperatura. Quando em operação, a pressão interna é maior do que 1,255 $\mathrm{MPa}$ (pressão de saturação para água a $190{ }^{\circ} \mathrm{C}$ ) [11], mas sempre menor que a máxima pressão admissível de trabalho do vaso.

Nas Figuras 1.1 e 1.2 são mostradas a vista lateral e frontal do VPCTVP-E-01. Pode ser visto a geometria do vaso, dimensões, o tampo utilizado e os tipos e posições dos bocais.

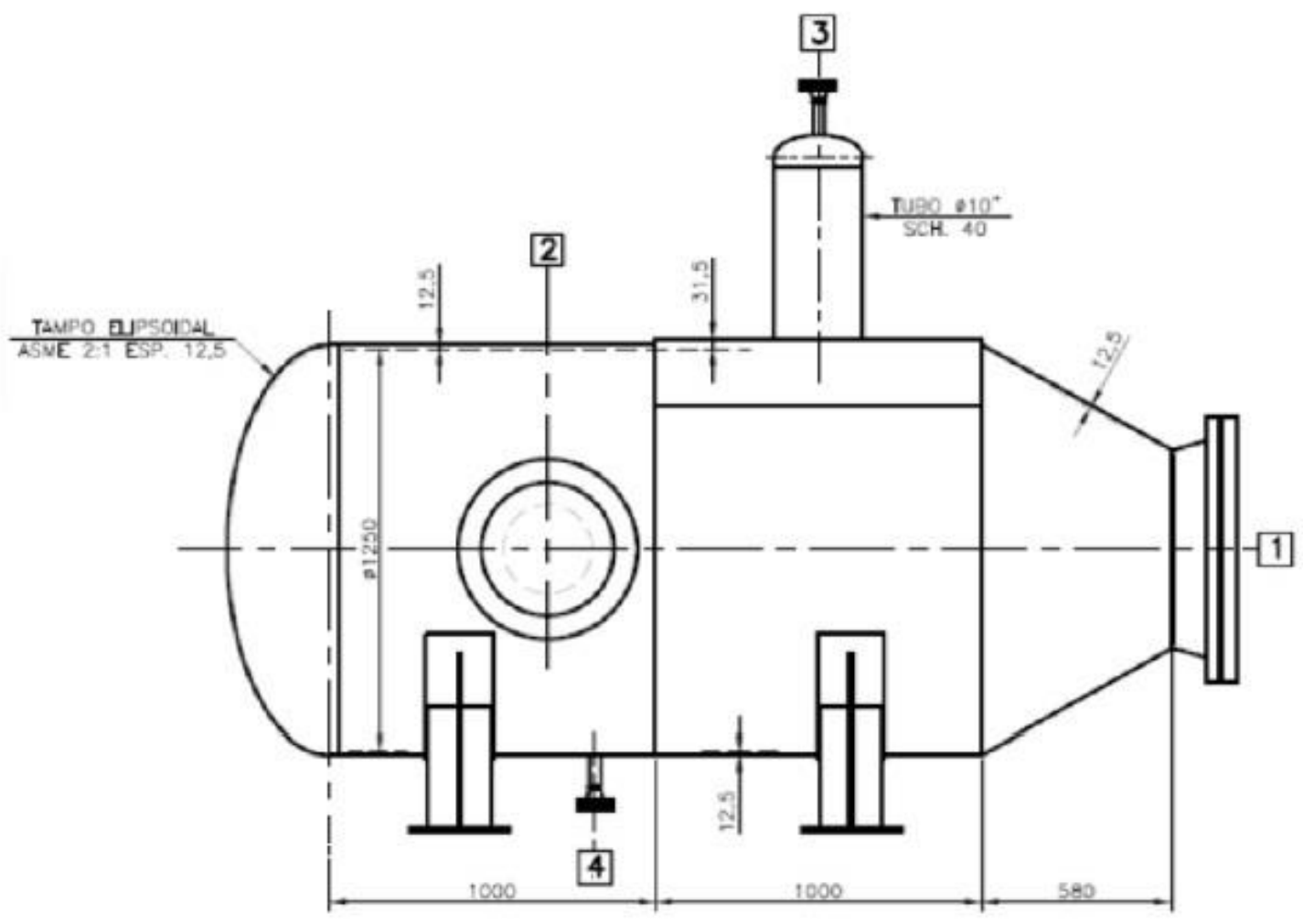

Figura 1.1: Desenho técnico do vaso VP-CTVP-E-01, parte 1 


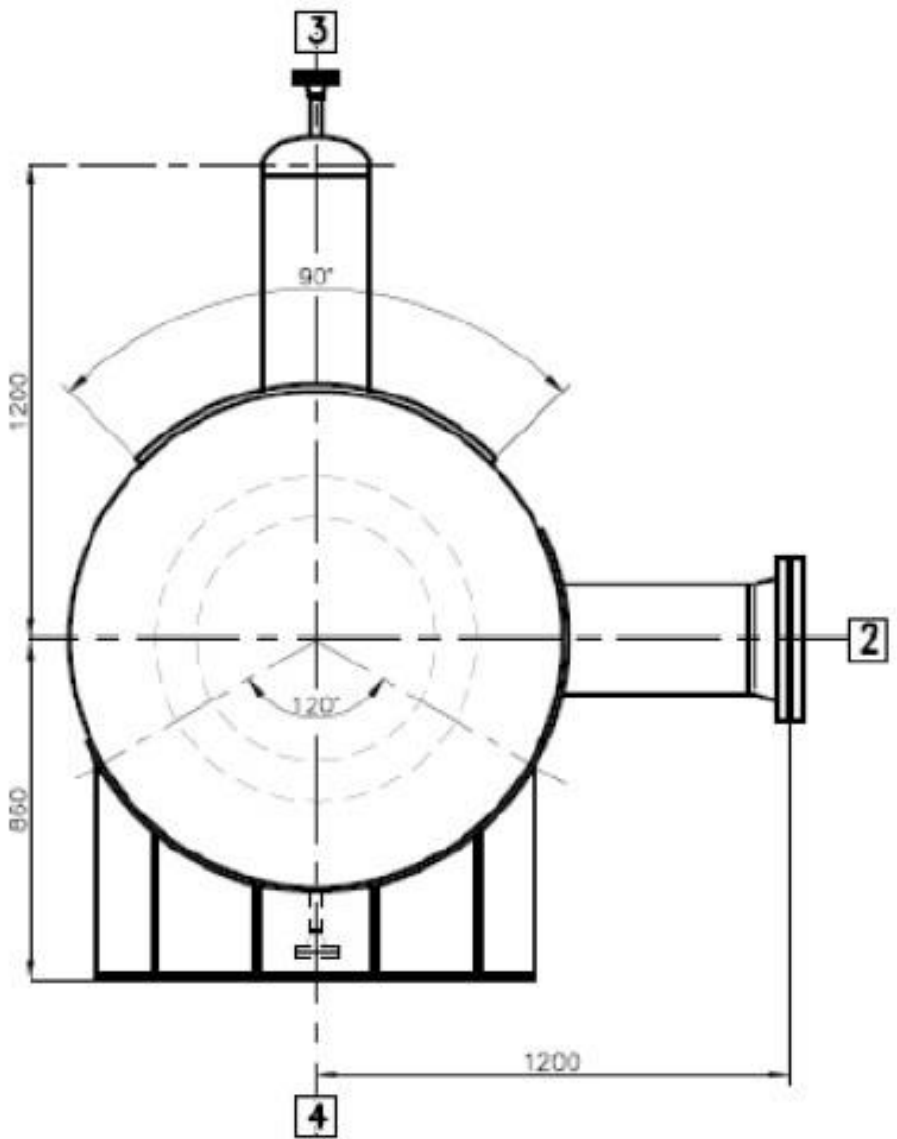

\begin{tabular}{|c|c|c|c|c|c|c|c|}
\hline \multicolumn{9}{|c|}{ BOCAIS } \\
\hline POS. & DIAM. & QT & CLASSE & TIPO & FACE & SCH. & SE KVIC O \\
\hline 1 & $24^{\prime \prime}$ & 1 & 150 & WN & RF & - & BOCA DE VISITA \\
\hline 2 & $10^{\prime \prime}$ & 1 & 150 & WN & RF & 40 & ENTRADA \\
\hline 3 & $1^{\prime \prime}$ & 1 & 150 & WN & RF & 40 & VENT. \\
\hline 4 & $1^{\prime \prime}$ & 1 & 150 & WN & RF & 40 & DRENO \\
\hline
\end{tabular}

Figura 1.2: Desenho técnico do vaso VP-CTVP-E-01, parte 2 
Na Figura 1.3 são indicadas todas as soldas que unem as diferentes partes do vaso, como as chapas, tampo, bocais, suportes e acessórios.

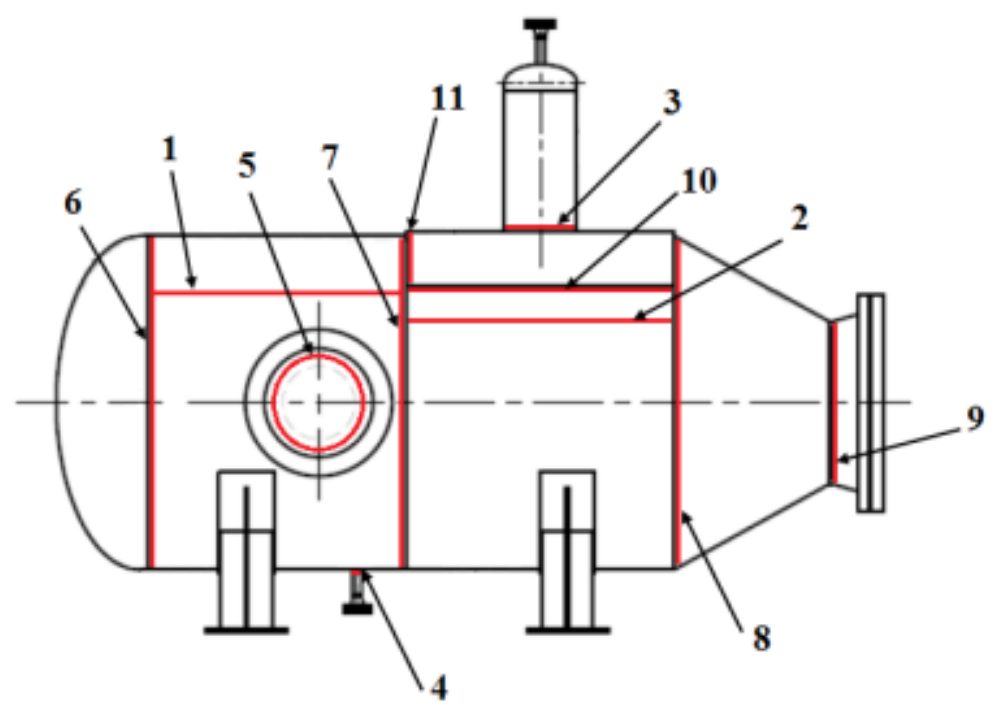

Figura 1.3: Numeração das juntas soldadas

A eficiência das juntas depende do seu tipo e da forma de inspeção especificada. Os valores destas eficiências [7] seguem na Tabela 1.1:

\begin{tabular}{|c|c|c|c|}
\hline $\mathbf{N}^{\mathbf{0}}$ da Junta & Categoria & Tipo de União & $\mathbf{E}$ \\
\hline 1 & A & Topo, duplo filete & 0.85 \\
\hline 2 & A & Topo, duplo filete & 0.85 \\
\hline 3 & B & Topo, único filete & 0.80 \\
\hline 4 & D & Canto & N/A \\
\hline 5 & D & Canto & N/A \\
\hline 6 & A & Topo, duplo filete & 0.85 \\
\hline 7 & A & Topo, duplo filete & 0.85 \\
\hline 8 & A & Topo, duplo filete & 0.85 \\
\hline 9 & C & Topo, duplo filete & 0.80 \\
\hline 10 & D & Canto & N/A \\
\hline 11 & A & Topo, duplo filete & 0.85 \\
\hline
\end{tabular}

Tabela 1.1: Eficiências das juntas soldadas 


\section{2}

\section{Objetivos}

O trabalho é divido em duas partes. Na primeira parte, o objetivo é fazer a análise estrutural estática do vaso de pressão considerado, usando a norma ASME, manuais de projeto e conceitos básicos de mecânica dos sólidos. A análise estrutural estática abrangerá conceitos como: corrosão; pressão de teste hidrostático e comparação entre a máxima pressão admissível de trabalho obtida pela Divisão 1 com aquela obtida pela Divisão 2 .

$\mathrm{Na}$ segunda parte, o objetivo é fazer uma análise de fadiga em toda a estrutura do vaso, incluindo os pontos críticos. A vida à fadiga deverá ser calculada de acordo com o modelo teórico apresentado pelo Método SN.

\section{3}

\section{Material didático utilizado}

O presente trabalho baseia-se primariamente na norma ASME de 2010; na teoria de fadiga [12] e no vaso de pressão VP-CTVP-E-01, que já foi projetado, e cujos dados básicos (e.g., geometria, construção e soldas) foram extraídos de [7].

Adicionalmente, foram utilizados manuais de projeto e análise de vasos de pressão. Todos estes manuais, que têm formato de livros, estão baseados na norma usada neste trabalho.

Também serviram como apoio outros projetos de graduação e mestrado sobre vasos de pressão [7], [8] e [9].

Finalmente, foram também usados para consulta livros de Mecânica dos Sólidos [13], tabelas de Termodinâmica [11] e sites de fabricantes de componentes para vasos de pressão [10]. 


\section{4}

\section{Divisão 1 x Divisão 2}

As regras para construção de vasos de pressão, segundo a Norma ASME, estão contidas na seção VIII e se dividem em três categorias: Divisão 1, Divisão 2 e Divisão 3. Neste trabalho, serão usadas as Divisões 1 e 2 . Por isso, é apresentado a seguir o resumo de cada uma, assim como suas principais diferenças:

Divisão 1: fornece regras para o projeto, fabricação, inspeção, teste e certificação de vasos de pressão que operam a pressões acima de $1 \mathrm{~atm}$.

Divisão 2: possui regras para construção e certificação de vasos de pressão que operam a pressões acima de 1 atm., usando métodos de análise. Usa tensões de projeto maiores que o da Divisão 1.

A diferença é que, enquanto na Divisão 1 se projeta por regras, na Divisão 2 se projeta por análise. As fórmulas são resultado de uma análise de tensões mais completa. Como consequência, os fatores de segurança são menores que o da Divisão 1, o que não compromete de forma alguma a segurança. Na verdade, é mais seguro se projetar pela Divisão 2, que é mais completa em termos da análise de tensões e dos correspondentes modos de falha. Tem ainda a vantagem de economizar material (já que se usa fatores de segurança mais racionais). O preço que se paga é o aumento de trabalho no projeto. 


\section{MATERIAL}

O ASTM A516 Gr 60 (ou SA-516 Gr. 60, pela norma ASME) é um aço com baixo teor de carbono $(\mathrm{C} \leq 0,3 \%)$. É comumente usado na construção de vasos de pressão que operam a temperaturas amenas e moderadas (-40 até $400{ }^{\circ} \mathrm{C}$ ). Suas principais propriedades mecânicas, nas temperaturas ambiente e de operação, foram extraídas de [3] e seguem nas Tabelas 2.1 e 2.2:

\begin{tabular}{|l|l|}
\hline \multicolumn{2}{|l|}{ Propriedades mecânicas do SA-516 Gr 60 à temperatura ambiente } \\
\hline Tensão mínima de escoamento & $220 \mathrm{MPa}$ \\
\hline Tensão mínima de ruptura & $415 \mathrm{MPa}$ \\
\hline Tensão admissível (Div. 1) & $118 \mathrm{MPa}$ \\
\hline Tensão admissível (Div. 2) & $147 \mathrm{MPa}$ \\
\hline Coeficiente de expansão térmica & $11,5 \times 10^{-6}\left(\mathrm{~mm} / \mathrm{mm} /{ }^{\circ} \mathrm{C}\right)$ \\
\hline Coeficiente de Poisson & 0,30 \\
\hline Módulo de elasticidade & $200 \mathrm{GPa}$ \\
\hline Densidade & $7750 \mathrm{~kg} / \mathrm{m}^{3}$ \\
\hline
\end{tabular}

Tabela 2.1: Propriedades mecânicas do SA-516 Gr 60 na temperatura ambiente

\begin{tabular}{|l|l|}
\hline \multicolumn{2}{|l|}{ Propriedades mecânicas do SA-516 Gr 60 a $190{ }^{\circ} \mathbf{C}$} \\
\hline Tensão mínima de escoamento & $189 \mathrm{MPa}$ \\
\hline Tensão mínima de ruptura & $414 \mathrm{MPa}$ \\
\hline Tensão admissível (Div. 1) & $118 \mathrm{MPa}$ \\
\hline Tensão admissível (Div. 2) & $126 \mathrm{MPa}$ \\
\hline $\begin{array}{l}\text { Coeficiente de expansão térmica } \\
\text { (instantâneo) }\end{array}$ & $13,8 \times 10^{-6}\left(\mathrm{~mm} / \mathrm{mm} /{ }^{\circ} \mathrm{C}\right)$ \\
\hline $\begin{array}{l}\text { Coeficiente de expansão térmica } \\
\text { (médio) }\end{array}$ & $12,7 \times 10^{-6}\left(\mathrm{~mm} / \mathrm{mm} /{ }^{\circ} \mathrm{C}\right)$ \\
\hline Coeficiente de Poisson & 0,30 \\
\hline Módulo de elasticidade & $192 \mathrm{GPa}$ \\
\hline Densidade & $7750 \mathrm{~kg} / \mathrm{m}^{3}$ \\
\hline
\end{tabular}

Tabela 2.2: Propriedades mecânicas do SA-516 Gr 60 a $190{ }^{\circ} \mathrm{C}$ 
Deve-se enfatizar que a norma ASME estabelece $370{ }^{\circ} \mathrm{C}$ como a temperatura limite para o SA-516 Gr 60. Este aço não deve ser usado para vasos de pressão que operem acima desta temperatura.

O coeficiente de expansão térmica médio é tomado a partir de $20{ }^{\circ} \mathrm{C}$, indo até a temperatura especificada [3].

Diferentemente das propriedades do aço, a tensão admissível não é medida por ensaios mecânicos, mas definida pela norma. Desta forma, além de variar com a temperatura, varia também com a Divisão usada nos cálculos.

A $190{ }^{\circ} \mathrm{C}$, a tensão admissível pela Divisão 2 é $6,8 \%$ maior do que a tensão admissível pela Divisão 1 (Tabela 2.2). Já na temperatura ambiente, esta diferença é de $25 \%$ (Tabela 2.1). Conforme discutido anteriormente, na Divisão 1 se projeta por regras, enquanto que na Divisão 2 se projeta por análise. Por isso que a tensão admissível para a Divisão 2 é maior. Uma análise de tensões mais completa tem como consequência coeficientes de segurança menores do que uma análise simplificada. 


\section{ANÁLISE ESTRUTURAL ESTÁTICA}

A análise estrutural a seguir se resumirá principalmente ao cálculo da MPAT (Máxima Pressão Admissível de Trabalho) pelas Divisões 1 e 2. A MPAT é a máxima pressão na qual um vaso pode operar durante sua vida de uma forma segura, i.e., garantida pela norma ASME. É calculada para suas principais partes. $\mathrm{O}$ valor que define a pressão limite é o menor obtido. Os cálculos são feitos considerando a espessura do costado na condição corroída. Os efeitos de carregamentos secundários (quando existirem) deverão ser levados em conta. Como as propriedades mecânicas variam com a temperatura, a MPAT deve ser calculada para cada temperatura designada.

O vaso será separado em três seções (suas três partes principais) e a MPAT será calculada para cada parte.

Ao final deste capítulo, será feita uma comparação entre os valores obtidos.

\section{1}

\section{Corrosão}

$\mathrm{Na}$ fase de projeto, ao serem determinadas as espessuras das partes, deve ser acrescida uma sobre-espessura de corrosão, que dependerá da taxa de corrosão esperada e da vida da estrutura. No caso do vaso já existir, quando então é calculada a máxima pressão de trabalho de operação, as espessuras nas fórmulas devem ser decrescidas da corrosão prevista. Para tal, o ideal é que seja conhecida a taxa de corrosão no local de operação. A profundidade do material corroído por ano, multiplicada pelo número de anos da vida de projeto, fornecerá o valor a ser subtraído da espessura. Para este trabalho, será suposta uma corrosão de 1,6 mm em 12 anos, que geralmente é um valor satisfatório [4]. 


\section{2}

\section{Cálculo da MPAT pela Divisão 1}

Notação:

P - Máxima Pressão Admissível de Trabalho

S - Tensão admissível do material

E - Eficiência da junta soldada

D - Diâmetro interno

$\mathrm{R}$ - Raio interno

$\mathrm{t}$ - Espessura da parede

$t_{c}$ - Espessura da parede corroída

\section{Casco cilíndrico:}

A fórmula que determina a MPAT para o casco cilíndrico [1] está baseada na tensão circunferencial que age no costado, que surge na presença de uma pressão interna manométrica. Como a tensão circunferencial solicita as juntas longitudinais, a eficiência usada (E) deve ser a correspondente à estas juntas. Tudo isso faz sentido, já que a tensão circunferencial é o dobro da longitudinal (Apêndice B). Só seria diferente se a eficiência das juntas circunferenciais fossem menos da metade da eficiência das juntas longitudinais, o que é bastante raro.

$$
P=\frac{S E t_{c}}{R+0,6 t_{c}}
$$

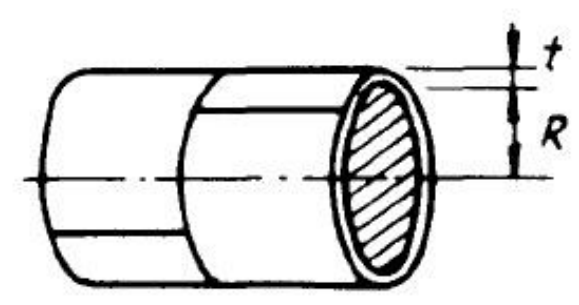

Figura 3.1: Casco Cilíndrico (3D)

Para as soldas do casco cilíndrico, $\mathrm{E}=0,85$ (Tabela 1.1) 
$\mathrm{R}=1250 / 2=625 \mathrm{~mm}$

$\mathrm{t}=12,5 \mathrm{~mm}$

$\mathrm{t}_{\mathrm{c}}=\mathrm{t}-\mathrm{ce}=12,5-1,6=10,9 \mathrm{~mm}$, onde ce significa 'corrosão estimada'.

$\mathrm{S}=118 \mathrm{MPa}$ (Tabela 2.1 ou 2.2)

Substituindo os valores na equação (3-1):

$\mathrm{P}=\frac{118 \times 0,85 \times 10,9}{625+0,6 \times 10,9}=1,731 \mathrm{MPa}$

Tampo elipsoidal:

A partir da referência [1]:

$$
P=\frac{2 S E t_{c}}{D+0,2 t_{c}}
$$

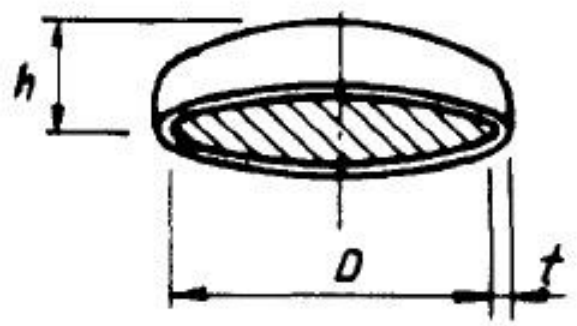

$$
h=\mathrm{D} / 4
$$

Figura 3.2: Tampo torisférico (3D)

$\mathrm{E}=0,8$ (Tabela 1.1)

$\mathrm{R}=625 \mathrm{~mm}$

$\mathrm{t}=12,5 \mathrm{~mm}$

$\mathrm{t}_{\mathrm{c}}=10,9 \mathrm{~mm}$

$\mathrm{S}=118 \mathrm{MPa}$

Então, $P=\frac{2 \times 118 \times 0,8 \times 10,9}{1250+0,2 \times 10,9}=1,643 \mathrm{MPa}$ 
Costado cônico:

De acordo com a referência [1]:

$$
\mathrm{P}=\frac{2 \mathrm{SEt} \mathrm{t}_{\mathrm{c}} \cos \alpha}{\mathrm{D}+1,2 \mathrm{t}_{\mathrm{c}} \cos \alpha}
$$

Deve se destacar que a equação (3-3) só é válida para $\alpha<30^{\circ}$.

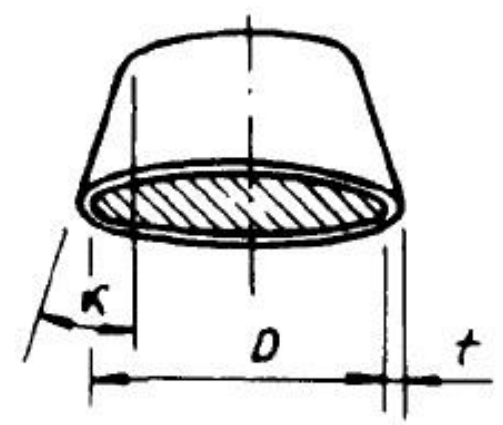

Figura 3.3: Casco cônico (3D)

Alpha $(\alpha)$ é a metade do ângulo central do setor circular do cone imaginário equivalente a esta seção.

$\alpha=28,91^{\circ}$

$\mathrm{E}=0,8$

$\mathrm{D}=1250 \mathrm{~mm}$

$\mathrm{t}_{\mathrm{c}}=10,9 \mathrm{~mm}$

$\cos \left(28,91^{\circ}\right)=0,87$

Desta forma, $\mathrm{P}=\frac{2 \times 118 \times 0,8 \times 10,9 \times 0,87}{1250+1,2 \times 10,9 \times 0,87}=1,419 \mathrm{MPa}$

Como a tensão admissível fornecida pela Divisão 1 é a mesma para ambas temperaturas (Tabelas 2.1 e 2.2), a MPAT também é igual. No entanto, se a 
temperatura de operação fosse mais alta, as tensões admissíveis seriam diferentes, e a MPAT teria que ser calculada para cada temperatura.

Não foi encontrada, na Divisão 1 da norma, regras para o cálculo da MPAT de bocais, nem mesmo equações de projeto que tivessem relação com a pressão interna. Por esta razão, somente será calculada a MPAT para o bocal pela Divisão 2.

$\mathrm{Na}$ Tabela 3.1 estão mostrados os valores calculados das MPAT segundo a Divisão 1. Portanto, a máxima pressão na qual o vaso pode operar é de 1,419 MPa.

\begin{tabular}{|c|c|}
\hline Parte do Vaso & $\begin{array}{l}\text { MPAT (em MPa) } \\
\text { (ASME Seção VIII Div. 1) }\end{array}$ \\
\hline Casco Cilíndrico & 1,731 \\
\hline Tampo Torisférico & 1,643 \\
\hline Costado Cônico & 1,419 \\
\hline Bocal &.-- \\
\hline
\end{tabular}

Tabela 3.1: Resultados das MPAT segundo a Divisão 1 


\section{3}

\section{Cálculo da PTH}

PTH é a Pressão de Teste Hidrostático. A norma ASME prevê que vasos de pressão que tenham sido projetados para resistir a pressões internas passem por um teste hidrostático [1]. Assim, após o vaso estar pronto (i.e., já com suas juntas soldadas e partes montadas), é feito um teste hidrostático, a fim de assegurar a estanqueidade. A pressão aplicada deve ser de no mínimo 1,3 vezes a máxima pressão admissível de trabalho multiplicada pela razão da tensão admissível do material do vaso na temperatura de teste pela tensão admissível do material do vaso na temperatura de projeto [1]. Tendo em vista que a tensão admissível não muda para esta faixa de temperatura, esta razão é igual a um, portanto:

$$
\begin{aligned}
& \mathrm{PTH}=1,3 \times \mathrm{MPAT} \times \frac{\mathrm{s}}{\mathrm{s} \prime} \\
& \mathrm{PTH}=1,3 \times 1,419 \times 1,0=1,845 \mathrm{MPa}
\end{aligned}
$$

Onde $S^{\prime}$ seria a tensão admissível do material na temperatura de operação.

Após o teste, deve ser feita uma inspeção em todas as juntas e conexões, com o intuito de identificar se há algum vazamento. Também devem ser checados os pontos onde há concentração de tensões, como por exemplo em volta do bocal, com o propósito de observar vazamento, ou ainda, se houve deformação excessiva naquele ponto. Enquanto esta inspeção é feita, a pressão interna no vaso deve ser maior ou igual à PTH dividida por 1,3 , i.e., $\mathrm{P}_{\text {inspeção }} \geq \mathrm{PTH} / 1,3$. 


\section{4}

\section{Cálculo da MPAT pela Divisão 2}

$\mathrm{Na}$ Divisão 2, só há fórmulas para o cálculo das espessuras, em função da pressão interna (ou seja, equações de projeto). No entanto, estas equações são facilmente inversíveis. Seguem os cálculos para as três seções e para o bocal, de acordo com a referência [2]:

Notação:

P - Máxima Pressão Admissível de Trabalho à temperatura ambiente $\mathrm{P}^{\prime}$ - Máxima Pressão Admissível de Trabalho a $190{ }^{\circ} \mathrm{C}$

$\mathrm{S}$ - Tensão admissível do material na temperatura ambiente

$\mathrm{S}^{\prime}$ - Tensão admissível do material a $190{ }^{\circ} \mathrm{C}$

E - Eficiência da junta soldada

D - Diâmetro interno

$\mathrm{R}$ - Raio interno

$\mathrm{t}$ - Espessura da parede

$t_{c}$ - Espessura da parede corroída

$\Theta$ - Temperatura

\section{Casco cilíndrico:}

$$
\begin{aligned}
\mathrm{t}_{\mathrm{c}} & =\frac{\mathrm{D}}{2}\left(\mathrm{e}^{\frac{\mathrm{P}}{\mathrm{SE}}}-1\right) \\
\therefore \mathrm{P} & =\mathrm{SE} \ln \left(\frac{2 \mathrm{t}_{\mathrm{c}}}{\mathrm{D}}+1\right)
\end{aligned}
$$

Para a temperatura ambiente:

$$
\mathrm{P}=147 \times 0,85 \times \ln \left(\frac{2 \times 10,9}{1250}+1\right)=2,160 \mathrm{MPa}
$$

A $190{ }^{\circ} \mathrm{C}$, o único valor que muda é o da tensão admissível do material. Assim, em vez de repetir a expressão toda, é mais simples multiplicar a pressão acima pela razão das tensões admissíveis:

$\mathrm{P}^{\prime}=\frac{126}{147} \mathrm{P}=1,851 \mathrm{MPa}$ 
Tampo elipsoidal:

A determinação da espessura mínima do tampo elipsoidal segue o parágrafo 4.3.7 da referência [2] e é um pouco mais complexa. As equações a serem usadas são as do parágrafo 4.3.6 da referência [2], com as seguintes substituições para $\mathrm{r}$ (raio da seção tórica) e L (raio da coroa calota esférica da região central do tampo):

$\mathrm{r}=\mathrm{D}\left(\frac{0,5}{\mathrm{k}}-0,08\right)$

$\mathrm{L}=\mathrm{D}(0,44 \mathrm{k}+0,02)$

Onde $k=\frac{D}{2 h}$

Para um maior esclarecimento destes parâmetros, seguem as Figuras 3.4 e 3.5, extraídas da referência [2]:

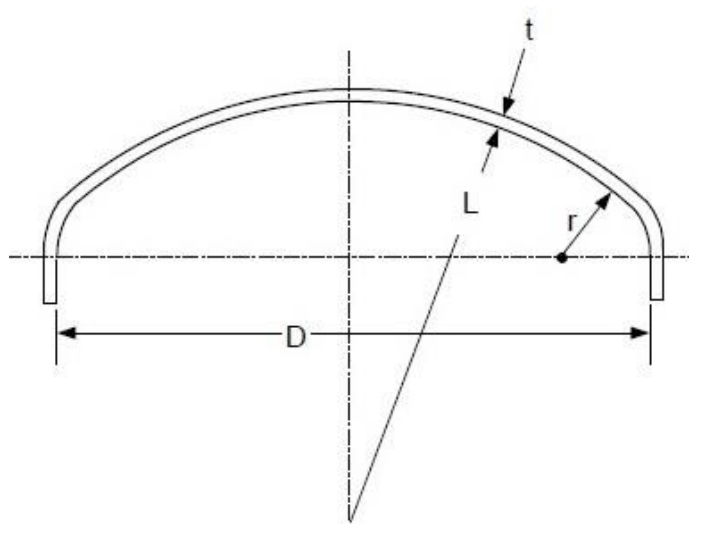

Figura 3.4: Tampo torisférico (2D)

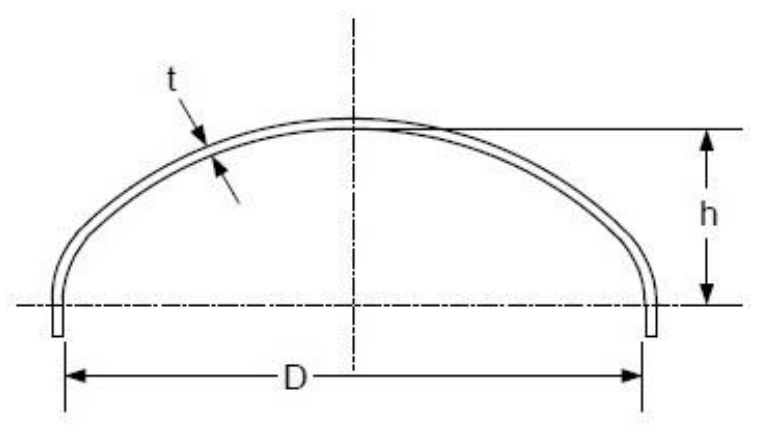

Figura 3.5: Tampo elipsoidal (2D) 
Da Figura 1.1, D (diâmetro interno) $=1250 \mathrm{~mm}$ e h (altura do tampo) $=\mathrm{D} / 4$ $=312,5 \mathrm{~mm}$. Esta é a relação entre $\mathrm{D}$ e $\mathrm{h}$ para um tampo elipsoidal da categoria ASME 2:1.

Substituindo os valores:

$$
\begin{aligned}
& \mathrm{k}=\frac{1250}{2 \times 312,5}=2 \\
& \mathrm{r}=1250\left(\frac{0,5}{2}-0,08\right)=212 \mathrm{~mm} \\
& \mathrm{~L}=1250(0,44 \times 2+0,02)=1125 \mathrm{~mm}
\end{aligned}
$$

Segundo o parágrafo $4.3 .7,1,7 \leq \mathrm{k} \leq 2,2$ para que as regras acima sejam válidas, o que é atendido.

Seguindo as orientações de 4.3.7, serão utilizadas as equações de 4.3.6, com as devidas substituições para $\mathrm{r}$ e L.

O parágrafo 4.3.6 estabelece que os cálculos sejam feitos em série, seguindo passos (STEP 1, STEP 2, etc). Alguns passos pedem que certos valores sejam testados antes de prosseguir, para que seja verificado se estão dentro de intervalos predeterminados. Caso estejam, o passo da série é executado; caso contrário, a norma determina que se projete por outro parágrafo.

O Passo 1 é determinar D, L, r e t.

Em seguida, no Passo 2, é necessário checar se as razões L/D; r/D e L/t estão entre os intervalos a seguir:

$0,7 \leq \mathrm{L} / \mathrm{D} \leq 1,0$

$\mathrm{r} / \mathrm{D} \geq 0,06$

$20 \leq \mathrm{L} / \mathrm{t} \leq 2000$

Substituindo os valores:

$$
\begin{aligned}
& \mathrm{L} / \mathrm{D}=1125 / 1250=0,9 \\
& \mathrm{r} / \mathrm{D}=212 / 1250=0,17 \\
& \mathrm{~L} / \mathrm{t}_{\mathrm{c}}=1125 / 10,9=103
\end{aligned}
$$


Como os valores acima atendem aos intervalos especificados, são calculadas as constantes geométricas, definidas pelo Passo 3:

$\beta_{\mathrm{th}}=\arccos \left[\frac{0,5 \mathrm{D}-\mathrm{r}}{\mathrm{L}-\mathrm{r}}\right]=\arccos \left[\frac{0,5 \times 1250-212}{1125-212}\right]=1,10 \mathrm{rad}$

$\varphi_{\mathrm{th}}=\frac{\sqrt{\mathrm{L} \times \mathrm{t}_{\mathrm{c}}}}{\mathrm{r}}=\frac{\sqrt{1125 \times 10,9}}{212}=0,52 \mathrm{rad}$

Como $\varphi_{\text {th }}<\beta_{\text {th }} \therefore \mathrm{R}_{\mathrm{th}}=\frac{0,5 \mathrm{D}-\mathrm{r}}{\cos \left[\beta_{\mathrm{th}}-\varphi_{\mathrm{th}}\right]}+\mathrm{r}=\frac{0,5 \times 1250-212}{\cos [1,10-0,52]}+212$

$\mathrm{R}_{\mathrm{th}}=706 \mathrm{~mm}$

No Passo 4 são calculados os coeficientes C1 e C2:

Como r/D > 0,08 $\therefore \mathrm{C}_{1}=0,692\left(\frac{\mathrm{r}}{\mathrm{D}}\right)+0,605=0,72$

e $C_{2}=1,46-2,6\left(\frac{r}{D}\right)=1,46-2,6 \times 0,17=1,02$

O Passo 5 determina o valor da pressão interna que causa a flambagem elástica da seção tórica:

$$
\begin{aligned}
& P_{\text {eth }}=\frac{\mathrm{C}_{1} \mathrm{E}_{\mathrm{t}} \mathrm{t}_{\mathrm{c}}{ }^{2}}{\mathrm{C}_{2} \mathrm{R}_{\mathrm{th}}\left(\frac{\mathrm{R}_{\mathrm{th}}}{2}-\mathrm{r}\right)} \\
& P_{\text {eth }}=\frac{0,72 \times 192 \times 10^{3} \times 10,9^{2}}{1,02 \times 706\left(\frac{706}{2}-212\right)}=162 \mathrm{MPa}
\end{aligned}
$$

sendo $E_{t} \mathrm{o}$ módulo de elasticidade na temperatura máxima de projeto (Tabela 2.2)

No Passo 6 é obtido o valor da pressão interna que resulta numa tensão máxima na seção tórica igual à tensão de escoamento do material:

$$
\mathrm{P}_{\mathrm{y}}=\frac{\mathrm{C}_{3} \mathrm{t}_{\mathrm{c}}}{\mathrm{C}_{2} \mathrm{R}_{\mathrm{th}}\left(\frac{\mathrm{R}_{\mathrm{th}}}{2 \mathrm{r}}-1\right)}
$$

Assumindo que a tensão admissível seja governada por propriedades independentes do tempo, então C3 = Sy (a tensão de escoamento do material na temperatura de projeto). 
Sendo $\theta=\theta$ amb:

$$
\mathrm{P}_{\mathrm{y}}=\frac{220 \times 10,9}{1,02 \times 706\left(\frac{706}{2 \times 212}-1\right)}=5 \mathrm{MPa}
$$

Sendo $\theta=190^{\circ} \mathrm{C}$ :

$$
\mathrm{P}_{\mathrm{y}}^{\prime}=\frac{189 \times 10,9}{1,02 \times 706\left(\frac{706}{2 \times 212}-1\right)}=4,30 \mathrm{MPa}
$$

No Passo 7 é calculado o valor da pressão interna que resulta em falha por flambagem da seção tórica:

$\mathrm{G}=\frac{\mathrm{P}_{\text {eth }}}{\mathrm{P}_{\mathrm{y}}}=\frac{149}{5}=29,8$ e $\mathrm{G}^{\prime}=\frac{\mathrm{P}_{\text {eth }}}{\mathrm{P}_{\mathrm{y}}}=\frac{149}{4,30}=34,6$

Para $G>1, P_{c k}=\left(\frac{0,77508 G-0,20354 G^{2}+0,019274 G^{3}}{1+0,19014 G-0,089534 G^{2}+0,0093965 G^{3}}\right) P_{y}$

Substituindo os valores na expressão:

$\mathrm{P}_{\mathrm{ck}}=2 \times 5=10 \mathrm{MPa}$ e $\mathrm{P}_{\mathrm{ck}}^{\prime}=2 \times 4,30=8,60 \mathrm{MPa}$

O Passo 8 determina a pressão admissível, baseada no mecanismo de falha por flambagem da seção tórica:

Para este passo, basicamente é aplicado um fator de segurança de 1,5

$$
\mathrm{P}_{\mathrm{ak}}=\frac{\mathrm{P}_{\mathrm{ck}}}{1,5}=\frac{10}{1,5}=6,66 \mathrm{MPa} \text { e } \mathrm{P}_{\mathrm{ak}}^{\prime}=\frac{\mathrm{P}_{\mathrm{ck}}}{1,5}=\frac{8,60}{1,5}=5,7 \mathrm{MPa}
$$

O Passo 9 calcula a pressão admissível baseada na ruptura da região central do tampo:

$$
\begin{aligned}
\mathrm{P}_{\mathrm{ac}} & =\frac{2 \mathrm{SE}}{\frac{\mathrm{L}}{\mathrm{t}_{\mathrm{c}}}+0,5} \\
P_{a c} & =\frac{2 \times 147 \times 0,85}{103,2+0,5}=2,410 \mathrm{MPa}
\end{aligned}
$$

Onde S (Tabelas 2.1 e 2.2) é a tensão admissível do material na temperatura especificada e E é a eficiência da junta soldada na região considerada (Tabela 1.1).

$$
\mathrm{P}_{\mathrm{ac}}^{\prime}=\frac{2 \times 126 \times 0,85}{103,2+0,5}=2,065 \mathrm{MPa}
$$


O Passo 10 determina a máxima pressão admissível:

$$
\mathrm{P}_{\mathrm{a}}=\min \left[\mathrm{P}_{\mathrm{ak}}, \mathrm{P}_{\mathrm{ac}}\right]
$$

$\mathrm{P}_{\mathrm{a}}=2,410 \mathrm{MPa}$

$\mathrm{P}_{\mathrm{a}}^{\prime}=2,065 \mathrm{MPa}$

Seção cônica:

De acordo com o parágrafo 4.3.4 da referência [2], a espessura mínima da seção cônica, Figura 3.6, deve ser calculada pela expressão:

$$
\mathrm{t}_{\mathrm{c}}=\frac{\mathrm{D}}{2 \cos \alpha}\left(\mathrm{e}^{\mathrm{P} / \mathrm{SE}}-1\right)
$$

Invertendo a equação (3-9) para P:

$$
\mathrm{P}=\mathrm{SE} \ln \left(\frac{2 \mathrm{t}_{\mathrm{c}} \cos \alpha}{\mathrm{D}}+1\right)
$$

$\mathrm{P}=147 \times 0,8 \times \ln \left(\frac{21,8 \times \cos \left(28.9^{0}\right)}{1250}+1\right)=1,782 \mathrm{MPa}$

$\mathrm{P}^{\prime}=\frac{126}{147} \mathrm{P}=1,527 \mathrm{MPa}$

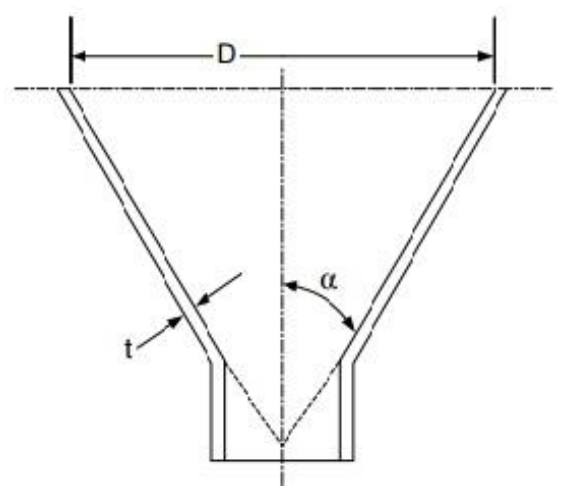

Figura 3.6: Seção cônica (2D) 


\section{$\underline{\text { Bocal }}$}

As MPAT, pela Divisão 2, para o Bocal, foram obtidas de acordo com a Subseção 4.5.5 (Radial Nozzle in a Cylindrical Shell) da referência [2]. Assim como o tampo elipsoidal, a MPAT é obtida através de uma receita, com 11 passos. Para que o leitor não fique entediado, os passos seguidos e cálculos feitos serão omitidos. O último passo contem as fórmulas que fornecem a MPAT. Estas fórmulas contêm parâmetros geométricos que foram obtidos a partir dos outros passos e a partir dos dados do vaso. As dimensões do tubo ANSI SCH. 40 - 10" usadas nos cálculos são catalogadas. Os valores obtidos foram de 2,500 MPa e 2,142 $\mathrm{MPa}$, para as temperaturas ambiente e de operação, respectivamente.

Nas Tabelas 3.2 e 3.3 estão mostrados os valores calculados das MPAT segundo a Divisão 2 para as temperaturas consideradas. Portanto, a máxima pressão na qual o vaso pode operar é de 1,527 MPa.

\begin{tabular}{|l|l|}
\hline Parte do Vaso & $\begin{array}{l}\text { MPAT (em MPa) } \\
\text { (ASME Seção VIII Div. 2) }\end{array}$ \\
\hline Casco Cilíndrico & 2,160 \\
\hline Tampo Torisférico & 2,410 \\
\hline Costado Cônico & 1,782 \\
\hline Bocal & 2,500 \\
\hline
\end{tabular}

Tabela 3.2: Resultados das MPAT para a temperatura ambiente segundo a Divisão 2

\begin{tabular}{|l|l|}
\hline Parte do Vaso & $\begin{array}{l}\text { MPAT } \\
\text { (ASME Seção VIII Div. 2) }\end{array}$ \\
\hline Casco Cilíndrico & 1,851 \\
\hline Tampo Torisférico & 2,065 \\
\hline Costado Cônico & 1,527 \\
\hline Bocal & 2,142 \\
\hline
\end{tabular}

Tabela 3.3: Resultados das MPAT para $190{ }^{\circ} \mathrm{C}$ segundo a Divisão 2 


\section{5}

\section{Comparação dos resultados}

Nas Tabelas 3.4 e 3.5, é apresentada a comparação dos resultados (em percentual) da Divisão 1 e da Divisão 2:

\begin{tabular}{|l|l|l|l|}
\hline Parte do Vaso & $\begin{array}{l}\text { MPAT (em MPa) } \\
\text { (ASME Seção } \\
\text { VIII Div. 1) }\end{array}$ & $\begin{array}{l}\text { MPAT (em MPa) } \\
\text { (ASME Seção } \\
\text { VIII Div. 2) }\end{array}$ & $\begin{array}{l}\text { Aumento } \\
\text { percentual da Div. } \\
\text { 1 para Div. 2 }\end{array}$ \\
\hline Casco Cilíndrico & 1,731 & 2,160 & $24,8 \%$ \\
\hline Tampo Torisférico & 1,643 & 2,410 & $46,7 \%$ \\
\hline Costado Cônico & 1,419 & 1,782 & $25,6 \%$. \\
\hline Bocal & ------------------- & 2,500 & ------------------- \\
\hline
\end{tabular}

Tabela 3.4: Aumento percentual da MPAT da Divisão 1 para Divisão 2, temperatura ambiente

\begin{tabular}{|c|c|c|c|c|}
\hline Parte do Vaso & \multicolumn{2}{|c|}{$\begin{array}{l}\text { MPAT (em MPa) } \\
\text { (ASME Seção } \\
\text { VIII Div. 1) }\end{array}$} & $\begin{array}{l}\text { MPAT (em MPa) } \\
\text { (ASME Seção } \\
\text { VIII Div. 2) }\end{array}$ & $\begin{array}{l}\text { Aumento } \\
\text { percentual da Div. } \\
1 \text { para Div. } 2\end{array}$ \\
\hline Casco Cilíndrico & 1,731 & 1,85 & & $6,9 \%$ \\
\hline Tampo Torisférico & 1,643 & 2,06 & & $25,7 \%$ \\
\hline Costado Cônico & 1,419 & 1,52 & & $7,6 \%$ \\
\hline Bocal & ------------------ & 2,12 & & --------------------------- \\
\hline
\end{tabular}

Tabela 3.5: Aumento percentual da MPAT da Divisão 1 para Divisão 2, temperatura de operação

Sendo o casco cônico a seção que limita a máxima pressão admissível de trabalho para este vaso, pode ser visto que quando se projeta pela Divisão 2, a MPAT aumenta em aproximadamente $25 \%$ para a temperatura ambiente e em cerca de $10 \%$ para a temperatura de operação. 


\section{ANÁLISE DA FADIGA NO VASO DE PRESSÃO}

É comum que vasos de pressão operem sob pressões e temperaturas que variam no tempo. Nessa situação, a aplicação repetida de cargas é a principal responsável pelo mecanismo de fadiga.

É assumido que o VP-CTVP-E-01 opera sob ciclos alternados de pressão, já que esta é uma condição usual para este tipo de equipamento. É também considerado que as variações de temperatura são lentas e não induzem tensões térmicas. Portanto, somente as tensões mecânicas devido à pressão são levadas em conta nas análises.

O vaso é assumido como estando completamente novo (caso contrário, deveria se conhecer o seu histórico de tensões). Além disso, por hipótese, não há qualquer trinca inicial, de forma que o estudo deste capítulo visa quantificar a vida da estrutura até o nascimento de uma trinca.

Tendo em vista, conforme será exposto na análise das tensões atuantes, que as mesmas são elásticas (inclusive nos pontos críticos), será utilizada a metodologia SN para prever a vida deste vaso. A vida aqui considerada é o número de ciclos de tensões a que o vaso pode ser submetido antes que seja iniciada uma macrotrinca. Ou seja, a fase de propagação da trinca não é levada em conta.

Finalmente, será checado pela norma ASME [2] se uma análise de fadiga se faz necessária. No entanto, não serão feitos cálculos de fadiga no vaso pela norma. 


\section{1}

\section{Avaliação da fadiga segundo a metodologia SN}

A metodologia $\mathrm{SN}$ visa prever os números de ciclos de tensão (que podem ser de gamas diferentes) que uma peça pode suportar até o aparecimento de uma trinca. Como é assumido material linear e elástico, o procedimento $\mathrm{SN}$ só deve ser usado quando as tensões que atuam nos pontos críticos da estrutura estão abaixo da tensão de escoamento do material nessas regiões. Tem boa precisão quando as gamas e máximos de tensões que atuam nos pontos críticos são baixos, prevendo bem vidas longas. Como o método SN não reconhece a presença de trincas, ele é usado para evitar ou prever a iniciação de trincas por fadiga.

O Método SN é divido em três etapas: 1) Quantificar a resistência à fadiga da peça ou estrutura; 2) Obter as tensões atuantes nos pontos críticos; c) Determinar o dano à fadiga.

As três etapas serão abordadas nos tópicos seguintes. A metodologia SN será primeiro aplicada às três seções principais do vaso: casco cilíndrico; seção cônica e tampo elipsoidal. Em seguida será refeito tudo para os 14 possíveis pontos críticos do vaso, que estão em regiões de descontinuidade, como nas soldas entre as placas ou nas juntas soldadas dos bocais. 


\subsection{1}

\section{Quantificando a resistência à fadiga da estrutura}

A seguir, será obtida a curva SN (ou curva de Wöhler) das três seções principais. Conforme será visto adiante em detalhes, a curva para as seções principais é a mesma (já que o material, temperatura e tipo de carregamento são os mesmos).

A relação parabólica mais usada para descrever a curva SN é:

$$
\mathrm{NS}_{\mathrm{F}}{ }^{\mathrm{B}}=\mathrm{C}
$$

$\mathrm{N}$ - Vida à fadiga em número de ciclos

$\mathrm{S}_{\mathrm{F}}$ - Resistência à fadiga

B - Expoente de Wöhler

C - Coeficiente de Wöhler

A equação (4-1) associa a resistência à fadiga com o correspondente número de ciclos, ou seja, $\mathrm{S}_{\mathrm{F}}$ é função de $\mathrm{N}\left(\mathrm{S}_{\mathrm{F}}(\mathrm{N})\right)$. Esta relação é obtida ajustando uma reta aos pontos plotados num gráfico log-log. B e C são constantes do material.

Para os aços, tipicamente se associa 1000 ciclos como uma vida curta e 1000000 de clicos como uma vida longa, sendo esta última geralmente associada como o limite de fadiga do aço.

Escolhendo os dois pontos extremos $\left(10^{\wedge} 3\right.$ e $10^{\wedge} 6$ ciclos $)$, pode ser obtida a equação da reta (lembrando que a curva $\mathrm{SN}$ é uma reta no gráfico $\log -\log )$. Desta forma, surge o sistema de equações:

$$
\left\{\begin{array}{l}
\mathrm{N}_{\mathrm{F}} \mathrm{S}_{\mathrm{F}}{ }^{\mathrm{B}}=\mathrm{C} \\
\mathrm{N}_{\mathrm{L}} \mathrm{S}_{\mathrm{L}}{ }^{\mathrm{B}}=\mathrm{C}
\end{array}\right.
$$

Sendo $\mathrm{N}_{\mathrm{F}}=10^{3}$ cliclos e $\mathrm{S}_{\mathrm{F}}$ a resistência à fadiga associada, e $\mathrm{N}_{\mathrm{L}}=$ $10^{6}$ ciclos e $S_{\mathrm{L}}$ a correspondente resistência. Dividindo a primeira equação pela segunda, o expoente de $\mathrm{S}$ pode ser explicitado:

$$
\mathrm{B}=\frac{\log \left(\mathrm{N}_{\mathrm{L}} / \mathrm{N}_{\mathrm{F}}\right)}{\log \left(\mathrm{S}_{\mathrm{F}} / \mathrm{S}_{\mathrm{L}}\right)}
$$


Segundo a referência [12], a resistência a fadiga dos aços em 1000 e $10^{\wedge} 6$ ciclos, e que tenham uma resistência à ruptura $\mathrm{Sr}<1400 \mathrm{MPa}$, pode ser estimada por:

$$
\begin{aligned}
& \mathrm{S}_{\mathrm{F}}\left(10^{3}\right)=\mathrm{k}_{\theta} \mathrm{k}_{\mathrm{e}} \times 0,76 \mathrm{~S}_{\mathrm{R}} \\
& \mathrm{S}_{\mathrm{L}}\left(10^{6}\right)=\mathrm{k}_{\mathrm{a}} \mathrm{k}_{\mathrm{b}} \mathrm{k}_{\mathrm{c}} \mathrm{k}_{\theta} \mathrm{k}_{\mathrm{e}} \times 0,5 \mathrm{~S}_{\mathrm{R}}
\end{aligned}
$$

Os $\mathrm{k}_{\mathrm{i}}$ acima são os fatores modificadores da resistência à fadiga do material, e são obtidos empiricamente. Conforme mostram as equações (43) e (4-4), para vidas curtas, os efeitos de acabamento superficial, $k_{a}$; de tamanho, $\mathrm{k}_{\mathrm{b}}$; e do tipo de carregamento, $\mathrm{k}_{\mathrm{c}}$, são desprezíveis. Só influenciam os efeitos de temperatura e de dispersão experimental. Já para o limite de fadiga, todos os efeitos podem influenciar de forma significativa. A seguir serão, abordados um a um, os $\mathrm{k}_{\mathrm{i}}$ para o vaso de pressão em estudo:

Fator de acabamento superficial, $\mathrm{k}_{\mathrm{a}}$. Para a escolha do $\mathrm{k}_{\mathrm{a}}$, foi considerado o processo de fabricação das chapas de aço que compõe o costado. A calandragem é um processo muito comum para curvar chapas de aço e foi o processo aqui considerado. No entanto, não há na literatura um fator de acabamento superficial para calandragem. O que mais se aproxima é o $\mathrm{k}_{\mathrm{a}}$ da laminação, já que a grosso modo, foi o processo mais semelhante. Como as chapas de aço deste vaso possuem todas uma espessura superiora a $3 \mathrm{~mm}$, a laminação a frio seria inviável. Portanto, se optou pelo fator da laminação a quente, que segundo a referência [12] é:

$$
k_{a}=56,1 \times S_{R}^{-0,719}
$$

A resistência à ruptura do SA-516 Gr. 60 é de $415 \mathrm{MPa}$ (Tabelas 2.1 e 2.2), logo:

$$
\mathrm{k}_{\mathrm{a}}=56,1(415)^{-0,719}=0,735
$$

$\mathrm{O}$ fator estocástico ou de confiabilidade, $\mathrm{k}_{\mathrm{e}}$, não foi levado em conta. Este fator visa compensar a dispersão dos dados. Como uma análise estatística foge do escopo deste trabalho, o mesmo não será considerado aqui.

Fator de tamanho, $\mathrm{k}_{\mathrm{b}}$. A finalidade deste fator é quantificar a influência do tamanho da peça (ou estrutura) no seu limite de fadiga. A referência [12] menciona que peças trabalhando sob cargas de tração/compressão 
alternadas devem usar um fator de tamanho $\mathrm{k}_{\mathrm{b}}=1$. O efeito do gradiente de tensões nulo deste caso é levado em conta por um fator de carregamento $\mathrm{k}_{\mathrm{c}}$, que será abordado a seguir.

Fator do tipo de carregamento, $\mathrm{k}_{\mathrm{c}}$. $\mathrm{O}$ fator de carga visa quantificar a diferença entre os limites de fadiga medidos sob tração/compressão e sob flexão rotativa. Para metais dúcteis, a referência [12] usa $\mathrm{k}_{\mathrm{c}}=0,9$ quando as cargas são axiais puras, sem fletores parasitas. Conforme será visto na análise de tensões, este é o tipo de carga que age em todas as três seções.

Fator de temperatura, $\mathrm{k}_{\theta}$. $\mathrm{O}$ fator $\mathrm{k}_{\theta}$ quantifica a variação do limite de fadiga da peça $S_{L}$ com a sua temperatura de trabalho $\theta_{t}$, quando os efeitos de fluência podem ser desprezados, ou seja, quando o teste é muito rápido ou quando $\theta_{\mathrm{t}}<\sim 0,3 \theta_{\mathrm{f}}$, a temperatura de fusão da liga metálica [12]:

$$
\mathrm{k}_{\theta}=\mathrm{S}_{\mathrm{L}}\left(\theta_{\mathrm{t}}\right) / \mathrm{S}_{\mathrm{L}}\left(\theta_{\mathrm{amb}}\right)
$$

$\mathrm{Na}$ ausência de resultados experimentais específicos, como $\mathrm{S}_{\mathrm{L}}$ é em geral proporcional a $S_{R}$, é proposto em [12] que:

$$
\mathrm{k}_{\theta}=\mathrm{S}_{\mathrm{R}}\left(\theta_{\mathrm{t}}\right) / \mathrm{S}_{\mathrm{R}}\left(\theta_{\mathrm{amb}}\right)
$$

Sendo $\theta_{\mathrm{t}}=190{ }^{\circ} \mathrm{C}$ e $\theta_{\mathrm{amb}} \cong 25^{\circ} \mathrm{C}, \mathrm{k}_{\theta}=414 / 415 \cong 1$ (ver Tabelas $2.1 \mathrm{e}$ 2.2).

Substituindo os valores dos $\mathrm{k}_{\mathrm{i}}$ nas equações (4-3) e (4-4), encontram-se as resistências à fadiga para $10^{3}$ e $10^{6}$ de ciclos:

$$
\begin{gathered}
\mathrm{S}_{\mathrm{F}}\left(10^{3}\right)=0,76 \times 415=315 \mathrm{MPa} \\
\mathrm{S}_{\mathrm{L}}\left(10^{6}\right)=0,735 \times 1 \times 0,9 \times 1 \times 0,5 \times 415=137 \mathrm{MPa}
\end{gathered}
$$

Agora, com a equação (4-2), é obtido o expoente de Wöhler:

$$
B=\frac{\log \left(10^{6} / 10^{3}\right)}{\log (315 / 137)}=\frac{3}{\log (315 / 137)}=8,3
$$

E, da equação (4-1), a constante de Wöhler pode ser calculada diretamente:

$\mathrm{ou}$

$$
\begin{aligned}
& C=10^{3} \times 315^{8,3}=5,44 \times 10^{23} \\
& C=10^{6} \times 137^{8,3}=5,43 \times 10^{23}
\end{aligned}
$$


Finalmente, a curva de Wöhler do vaso:

$$
\mathrm{NS}^{8,3}=5,44 \times 10^{23}
$$

\subsection{2}

\section{Análise das tensões atuantes}

Neste tópico, serão calculadas as tensões principais para as três seções do vaso: costado; seção cônica e tampo torisférico. Em seguida, será usado o critério de Tresca para combinar as tensões principais. A partir das tensões combinadas, serão obtidas as tensões alternadas e médias, que surgem devido à variação na pressão interna. Finalmente, serão obtidas por Goodman as tensões alternadas equivalentes.

A geometria do vaso de pressão deste trabalho pode ser replicada pela Figura 4.1 [7], que mostra as variáveis dimensionais que serão usadas nos cálculos das tensões principais:

$\sigma_{1}$

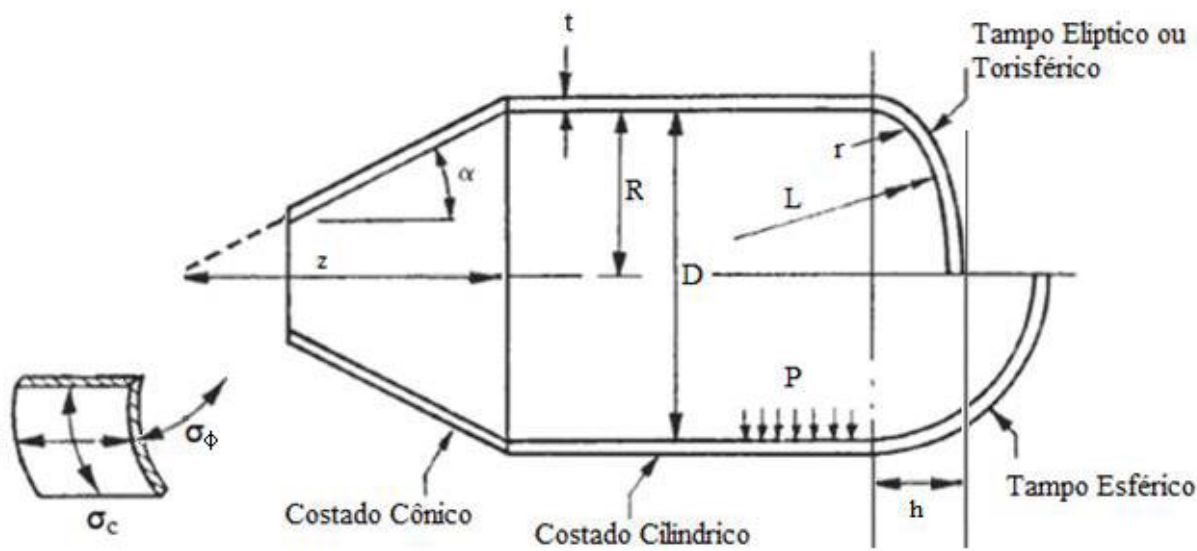

Figura 4.1: Principais variáveis dimensionais do vaso de pressão [7]

Quando na temperatura ambiente, será assumida uma pressão interna igual a atmosférica $(\sim 0,101 \mathrm{MPa})$. Já para a pressão de operação (quando a temperatura do vaso de pressão é de $190{ }^{\circ} \mathrm{C}$ ), a pressão interna é superior a 1,255 $\mathrm{MPa}$ (pressão de saturação da água a $190{ }^{\circ} \mathrm{C}$ ) + 0,0124 (componente hidrostática da coluna d'água) $=1,2674 \mathrm{MPa}$. Visando garantir que a água 
esteja sempre em estado líquido, será adotada uma pressão interna de 1,3 MPa (a pressão interna não pode ultrapassar a MPAT).

Quando há variação significativa de temperatura deve-se também levar em conta as tensões térmicas. Essas tensões surgem quando há restrições aos deslocamentos (ou deformações) causados por variação de temperatura. Por exemplo, vasos de pressão que de alguma forma estejam restritos à livre expansão por apoios externos, sofrerão tensões compressivas ao serem aquecidos, pois os apoios irão impedir (total ou parcial) o aumento de volume que ocorreria normalmente. Outra forma em que apareceriam tensões térmicas seria se a parede do vaso fosse grossa e/ou se o transiente de temperatura fosse acentuado. Neste caso haveriam tensões térmicas ao longo da espessura, na direção radial.

Para o vaso de pressão deste trabalho são feitas as seguintes considerações para que as tensões térmicas não sejam incluídas nas análises:

a) Não há restrição à livre deformação por apoios externos. Nesse caso o costado é apoiado sobre as selas, e não soldado e não está sendo levado em conta o tubo acoplado ao bocal 2 (Figura 1.2);

b) Os gradientes de temperatura (que ocorrem quando $\theta$ varia de 25 a $190{ }^{\circ} \mathrm{C}$, ou vice-versa) são baixos. Além disso, as paredes deste vaso são finas, o que faz com que seja atingida rapidamente uma temperatura uniforme ao longo da espessura.

O critério de Tresca estabelece que:

$$
\sigma_{\mathrm{T}}=\sigma_{1}-\sigma_{3}
$$

onde $\sigma_{1}, \sigma_{2}$ e $\sigma_{3}$ são as tensões principais e $\sigma_{1}>\sigma_{2}>\sigma_{3}$.

\section{Costado:}

No costado cilíndrico, as três tensões principais são (Apêndice B):

$$
\sigma_{1}=\sigma_{c}=\frac{P R}{t_{c}} ; \quad \sigma_{2}=\sigma_{1}=\frac{P R}{2 t_{c}} \text { e } \sigma_{3}=-P
$$


Portanto, a tensão combinada, por Tresca, é de:

$$
\sigma_{\mathrm{T}}=\sigma_{1}-\sigma_{3}=\frac{\mathrm{PR}}{\mathrm{t}_{\mathrm{c}}}-(-\mathrm{P})=\mathrm{P}\left(\mathrm{R} / \mathrm{t}_{\mathrm{c}}+1\right)
$$

Para $\mathrm{P}=0,101 \mathrm{MPa}$ :

$$
\sigma_{\mathrm{T}}=0,101(636 / 10,9+1)=6 \mathrm{MPa}
$$

Já quando $\mathrm{P}=1,300 \mathrm{MPa}$ :

$$
\sigma_{\mathrm{T}}=1,3(636 / 10,9+1) \cong 77,2 \mathrm{MPa}
$$

O raio usado nos cálculos acima é o externo $(625+10,9)$, já que gera previsões mais seguras (Apêndice B).

A variação temporal da pressão interna irá causar uma gama de tensão, cujo mínimo é de $6 \mathrm{MPa}$ e máximo de $77,2 \mathrm{MPa}$, respectivamente. Os ciclos desta gama de tensão $(6 \rightarrow 77,2 \rightarrow 6 \rightarrow 77,2)$ estão em fase com os ciclos de pressão $(0,101 \rightarrow 1,3 \rightarrow 0,101 \rightarrow 1,3)$.

A tensão alternada e média são obtidas por:

$$
\sigma_{a}=\frac{\Delta \sigma}{2}=\frac{\sigma_{\operatorname{máx}}-\sigma_{\min }}{2} \text { e } \sigma_{m}=\frac{\sigma_{\operatorname{máx}}+\sigma_{\min }}{2}
$$

Que para o mínimo e máximo dos ciclos de tensão considerado, valem: $\sigma_{\mathrm{a}}=\frac{77,2-6}{2}=35,6 \mathrm{MPa}$ e $\sigma_{\mathrm{m}}=\frac{77,2+6}{2}=41,6 \mathrm{MPa}$.

A tensão alternada equivalente, por Goodman, é dada por [12]:

$$
\begin{aligned}
& \sigma_{\text {aeq }}=\frac{\sigma_{\mathrm{a}}}{1-\sigma_{\mathrm{m}} / \mathrm{S}_{\mathrm{R}}} \\
& \therefore \sigma_{\text {aeq }}=\frac{35,6}{1-\frac{41,6}{415}}=39,6 \mathrm{MPa}
\end{aligned}
$$

Onde $S_{R}$ é a resistência à ruptura por tração do material, que para o SA-516 Gr 60 vale $415 \mathrm{MPa}$ (Tabelas 2.1 e 2.2).

Portanto, $\sigma_{\text {aeq }}=39,6 \mathrm{MPa}$. 
Deve ser salientado que, geralmente, em projetos e análises de fadiga, são aplicados fatores de segurança. Estes fatores multiplicam as tensões alternada e média. Por exemplo, para um fator de segurança contra fadiga $\varphi_{F}=1,5$, a tensão alternada equivalente seria calculada utilizando $1,5 \sigma_{\mathrm{a}}$ e $1,5 \sigma_{\mathrm{m}}$ em vez de somente $\sigma_{\mathrm{a}}$ e $\sigma_{\mathrm{m}}$. No entanto, conforme será visto na Subseção 4.1.4, as menores vidas virão dos pontos críticos. Não há necessidade de aplicar fatores de segurança em todas as seções e pontos analisados, mas somente onde for obtida a menor das vidas, re-calculando em seguida esta última com o emprego de $\varphi_{F}$, já que é esta que ditará a vida do vaso. Conforme será visto na Subseção 4.1.4, fatores de segurança contra fadiga, quando usados em soldas, "entram" multiplicando $\Delta \sigma$.

É importante que também seja calculada a tensão alternada equivalente dos ciclos associados aos testes hidrostáticos. Conforme será visto na Subseção 4.1.3, é considerado para este trabalho a aplicação de 5 TH. Da Seção 3.3 tem-se que a PTH (Pressão de Teste Hidrostático) é de $1,845 \mathrm{MPa}$ e, portanto,

$\therefore \sigma_{\mathrm{T}}=\mathrm{P}\left(\frac{\mathrm{R}}{\mathrm{t}_{\mathrm{c}}}+1\right) \cong 1,85\left(\frac{636}{10,9}+1\right) \cong 110 \mathrm{MPa}$

Desta forma, os ciclos de tensão associados aos testes hidrostáticos terão um mínimo de 6 e um máximo de $110 \mathrm{MPa}$. Portanto, a tensão alternada será de $52 \mathrm{MPa}$, a média de $58 \mathrm{MPa}$ e a tensão alternada equivalente (por Goodman) é de $60 \mathrm{MPa}$.

Seção cônica:

Segundo [7], as tensões principais que agem no costado cônico são:

$$
\sigma_{1}=\sigma_{\mathrm{c}}=\frac{\mathrm{P} \times \mathrm{z} \times \tan \alpha}{\mathrm{t}_{\mathrm{c}}} \quad \sigma_{2}=\sigma_{1}=\frac{\sigma_{\mathrm{c}}}{2} \quad \sigma_{3}=\sigma_{\varphi}=-\mathrm{P}
$$

Substituindo $\alpha=28,9^{\circ}$ (Seção 3.2), z = $842 \mathrm{~mm} \mathrm{[7]} \mathrm{e} t_{c}=10,9 \mathrm{~mm}$ na equação para $\sigma_{1}$ (Equação 4-12), são obtidas as seguintes tensões:

Quando P = 1,3 MPa:

$$
\sigma_{1}=55,4 \mathrm{MPa}, \sigma_{2}=27,7 \mathrm{MPa} \text { e } \sigma_{3}=-1,3 \mathrm{MPa}
$$




$$
\therefore \sigma_{\mathrm{T}}=\sigma_{1}-\sigma_{3}=55,4-(-1,3)=56,7 \mathrm{MPa}
$$

Se $\mathrm{P}=0,101 \mathrm{MPa}$ :

$$
\begin{gathered}
\sigma_{1}=4,3 \mathrm{MPa}, \sigma_{2}=2,15 \mathrm{MPa} \text { e } \sigma_{3}=-0,101 \mathrm{MPa} \\
\therefore \sigma_{\mathrm{T}} \cong 4,4 \mathrm{MPa}
\end{gathered}
$$

E para a PTH $(\mathrm{P}=1,85 \mathrm{MPa})$ :

$$
\begin{gathered}
\sigma_{1}=78,8 \mathrm{MPa}, \sigma_{2}=39,4 \mathrm{MPa} \text { e } \sigma_{3}=-1,85 \\
\therefore \sigma_{\mathrm{T}} \cong 80,7 \mathrm{MPa}
\end{gathered}
$$

Como resultado aparecem duas gamas de tensão: a de operação, $\Delta \sigma_{\text {op }}(4,4 \rightarrow 56,7 \rightarrow 4,4)$ e a do teste hidrostático, $\Delta \sigma_{\mathrm{TH}}(4,4 \rightarrow$ $80,7 \rightarrow 4,4)$.

Para a gama de tensão de operação: $\sigma_{\mathrm{a}}=26,2 \mathrm{MPa}, \sigma_{\mathrm{m}}=30,6 \mathrm{MPa}$ e $\sigma_{\text {aeq }}=28,3 \mathrm{MPa}$.

Já para a gama de tensão do teste hidrostático: $\sigma_{\mathrm{a}}=38,2 \mathrm{MPa}, \sigma_{\mathrm{m}}=42,6$ $\mathrm{MPa}$ e $\sigma_{\text {aeq }}=42,6 \mathrm{MPa}$.

Tampo elipsoidal:

Para o tampo torisférico, as tensões principais são [7]:

$$
\sigma_{1}=\sigma_{2}=\sigma_{c}=\sigma_{\varphi}=\frac{\mathrm{PR}^{2}}{2 \times \mathrm{t}_{\mathrm{c}} \times \mathrm{h}} \text { e } \sigma_{3}=\sigma_{1}=-\mathrm{P}
$$

Pela Figura 4.1, fica claro que as tensões circunferencial, longitudinal e normal estão em outra disposição, já que mudaram de plano.

Substituindo $h \cong 312 \mathrm{~mm}$ (Seção 3.4) e as demais variáveis nas equações para $\sigma_{1}$ e $\sigma_{3}$, é obtido:

Para $\mathrm{P}=0,101 \mathrm{MPa}$ :

$\sigma_{1}=\sigma_{2}=5,8 \mathrm{MPa} \mathrm{e} \sigma_{3}=-0,101 \mathrm{MPa}$

$\sigma_{\mathrm{T}}=\sigma_{1}-\sigma_{3}=5,8-(-0,101) \cong 6 \mathrm{MPa}$

Para $\mathrm{P}=1,3 \mathrm{MPa}$ 
$\sigma_{1}=\sigma_{2}=74,7 \mathrm{MPa}$ e $\sigma_{3}=-1,3 \mathrm{MPa}$

$\sigma_{\mathrm{T}}=76 \mathrm{MPa}$

E quando $\mathrm{P}=1,85 \mathrm{MPa}$

$\sigma_{1}=\sigma_{2} \cong 106 \mathrm{MPa} \mathrm{e} \sigma_{3}=-1,85 \mathrm{MPa}$

$\sigma_{\mathrm{T}} \cong 108 \mathrm{MPa}$

No tampo torisférico, as gamas de tensão serão: $\Delta \sigma_{o p}(6 \rightarrow$ $76 \rightarrow 6)$ e $\Delta \sigma_{T H}(6 \rightarrow 108 \rightarrow 6)$.

Para a gama de tensão de operação: $\sigma_{\mathrm{a}}=35 \mathrm{MPa}, \sigma_{\mathrm{m}}=41 \mathrm{MPa}$ e $\sigma_{\text {aeq }}=$ 38,8 MPa.

Já para a gama de tensão do teste hidrostático: $\sigma_{\mathrm{a}}=51 \mathrm{MPa}, \sigma_{\mathrm{m}}=57$ $\mathrm{MPa}$ e $\sigma_{\text {aeq }}=59,1 \mathrm{MPa}$.

Antes de encerrar o tópico da análise de tensões, é importante destacar que todas as tensões principais (para as três seções e três pressões) e as tensões alternadas equivalentes correspondentes são menores do que a tensão de escoamento do SA-516 Gr. 60. Logo, o método SN fornecerá resultados com precisão satisfatória.

\subsection{3}

\section{Acúmulo do dano à fadiga}

Finalmente, com a curva de Wöhler do material e os estados de tensão definidos, é obtido o número de ciclos que o vaso de pressão pode suportar. A vida $\mathrm{N}$ está associada à tensão alternada equivalente e às constantes de Wöhler através da relação:

$$
\mathrm{N} \sigma_{\mathrm{aeq}}{ }^{\mathrm{B}}=\mathrm{C}
$$

Sendo esta análoga à equação (4-1), com tensão no lugar de resistência. 
Com todas as informações obtidas até agora, o número de ciclos $(\mathrm{N})$ até o início de uma trinca pode ser obtido através da relação (4-14). $\mathrm{N}$ deve ser calculado para cada seção. A vida do vaso será o número de ciclos que a seção menos resistente à fadiga suporta. No entanto, deve-se antes computar o dano à fadiga causado pelos testes hidrostáticos. É comum que seja realizado somente um TH durante toda a vida de um vaso de pressão. Evidentemente que o número de TH depende do tipo de vaso e aplicação. Neste trabalho, será assumido $5 \mathrm{TH}$, uma hipótese razoavelmente conservadora.

Cada um dos eventos da carga $\left(\sigma_{a i}, \sigma_{m i}\right)$ causa um dano $D_{i}$, que pode ser quantificado por [12]:

$$
\mathrm{D}_{\mathrm{i}}=\frac{\mathrm{n}_{\mathrm{i}}}{\mathrm{N}_{\mathrm{i}}}
$$

Onde $\mathrm{n}_{\mathrm{i}}$ é o número de ciclos em que o i-ésimo evento atuou na estrutura e $\mathrm{N}_{\mathrm{i}}$ o número de ciclos que a estrutura duraria se apenas aquele evento nela atuasse.

A regra de Miner prevê falha quando a soma dos danos igualar o dano crítico, definido por $\mathrm{D}=1$. Ou seja, quando:

$$
\sum \frac{\mathrm{n}_{\mathrm{i}}}{\mathrm{N}_{\mathrm{i}}}=1
$$

Seguindo o procedimento previamente descrito, será calculada a vida de cada seção:

\section{Casco cilíndrico:}

O número de Testes Hidrostáticos que o casco pode suportar é obtido através da equação (4-14):

$$
\begin{gathered}
\mathrm{N}_{\mathrm{TH}}=\frac{\mathrm{C}}{\sigma_{\mathrm{aeq}}{ }^{\mathrm{B}}}=\frac{5,44 \times 10^{23}}{60^{8,3}}=9,48 \times 10^{8} \text { ciclos } \\
\mathrm{D}_{\mathrm{TH}}=\frac{\mathrm{n}_{\mathrm{TH}}}{\mathrm{N}_{\mathrm{TH}}}=\frac{5}{9,48 \times 10^{8}}=5,27 \times 10^{-9}
\end{gathered}
$$


Neste caso, o dano causado pela aplicação dos TH é muito baixo (praticamente zero). No entanto, é importante deixar claro que isto poderia ser diferente.

Para os ciclos de operação, o vaso suportaria:

$$
\mathrm{N}_{\mathrm{op}}=\frac{\mathrm{C}}{\sigma_{\mathrm{aeq}}{ }^{\mathrm{B}}}=\frac{5,44 \times 10^{23}}{39,6^{8,3}}=2,98 \times 10^{10} \text { ciclos }
$$

Portanto, o número de ciclos de operação que o vaso pode suportar é de:

$$
\mathrm{n}_{\mathrm{op}}=\mathrm{N}_{\mathrm{op}}\left(1-\mathrm{D}_{\mathrm{TH}}\right)=2,98 \times 10^{10} \text { ciclos }
$$

O que é uma vida infinita. Fica claro que o dano dos testes hidrostáticos é realmente insignificante para o presente caso, uma vez que $\mathrm{n}_{\mathrm{op}}=\mathrm{N}_{\mathrm{op}}$.

Costado cônico:

$$
\begin{gathered}
\mathrm{N}_{\mathrm{TH}}=\frac{\mathrm{C}}{\sigma_{\mathrm{aeq}}{ }^{\mathrm{B}}}=\frac{5,44 \times 10^{23}}{42,6^{8,3}}=1,62 \times 10^{10} \text { ciclos } \\
\mathrm{D}_{\mathrm{TH}}=\frac{\mathrm{n}_{\mathrm{TH}}}{\mathrm{N}_{\mathrm{TH}}}=\frac{5}{1,62 \times 10^{10}}=3,09 \times 10^{-10} \approx 0
\end{gathered}
$$

Como este é novamente um dano completamente desprezível, a vida do costado será calculada considerando que o mesmo seja virgem (i.e., ainda não tenha sido exposto a nenhum carregamento):

$$
\mathrm{n}_{\mathrm{op}}=\mathrm{N}_{\mathrm{op}}=\frac{\mathrm{C}}{\sigma_{\mathrm{aeq}} \mathrm{B}^{\mathrm{B}}}=\frac{5,44 \times 10^{23}}{28,3^{8,3}}=4,85 \times 10^{11} \text { ciclos }
$$

\section{Tampo torisférico:}

$$
\begin{gathered}
\mathrm{N}_{\mathrm{TH}}=\frac{\mathrm{C}}{\sigma_{\mathrm{aeq}}{ }^{\mathrm{B}}}=\frac{5,44 \times 10^{23}}{59,1^{8,3}}=1,075 \times 10^{9} \text { ciclos } \\
\mathrm{D}_{\mathrm{TH}}=\frac{\mathrm{n}_{\mathrm{TH}}}{\mathrm{N}_{\mathrm{TH}}}=\frac{5}{1,075 \times 10^{9}}=4,65 \times 10^{-9} \approx 0 \\
\therefore \mathrm{n}_{\mathrm{op}}=\mathrm{N}_{\mathrm{op}}=\frac{\mathrm{C}}{\sigma_{\mathrm{aeq}}{ }^{\mathrm{B}}}=\frac{5,44 \times 10^{23}}{38,8^{8,3}}=3,53 \times 10^{10} \mathrm{ciclos}
\end{gathered}
$$


Na Tabela 4.1, é apresentada a vida obtida para cada seção:

\begin{tabular}{|l|c|}
\hline \multicolumn{2}{|c|}{ Vida à Fadiga nas Seções do Vaso } \\
\hline Seção & Vida $\left(\mathrm{n}^{\mathbf{o}}\right.$ de ciclos $)$ \\
\hline Casco cilíndrico & $2,98 \times 10^{10}$ \\
\hline Costado cônico & $4,85 \times 10^{11}$ \\
\hline Tampo torisférico & $3,53 \times 10^{10}$ \\
\hline
\end{tabular}

Tabela 4.1: Vidas à fadiga obtidas nas seções do vaso

Portanto, o número de ciclos de pressão $(0,101 \rightarrow 1,3 \rightarrow 0,101) \mathrm{MPa}$ a que o vaso é submetido até a iniciação de uma macrotrinca por fadiga ( 1 a $2 \mathrm{~mm}$ ) é limitado pelo costado cilíndrico, sendo, portanto, de $2,98 \times 10^{10}$ ciclos. Como a vida infinita para os aços é definida em algo entre $10^{6} \mathrm{e}$ $10^{7}$ ciclos, a vida estimada anteriormente é, de longe, infinita. Mesmo que o número de ciclos encontrado fosse dividido por 1000, esta vida ainda seria infinita (para aços).

A análise feita anteriormente, apesar de importante, não leva à previsão correta da vida do vaso de pressão. Isto porque a trinca irá nascer em algum dos pontos críticos da estrutura, que estão localizados em suas juntas soldadas. A seguir, será calculada a vida dos quatro pontos mais críticos, a fim de obter a previsão correta do número de ciclos de pressão a que o vaso pode ser submetido.

\subsection{4}

\section{Análise do dano à fadiga nos pontos críticos}

Em [7] foram identificados 14 prováveis pontos críticos no vaso de pressão. Todos os 14 pontos estão em regiões de descontinuidade, como nas soldas entre as placas ou nas juntas soldadas dos bocais. Na Figura 4.2 estão mostrados estes pontos, que quando comparados à Figura 1.3, podem ser diretamente localizados nas 11 juntas da estrutura como um todo. 


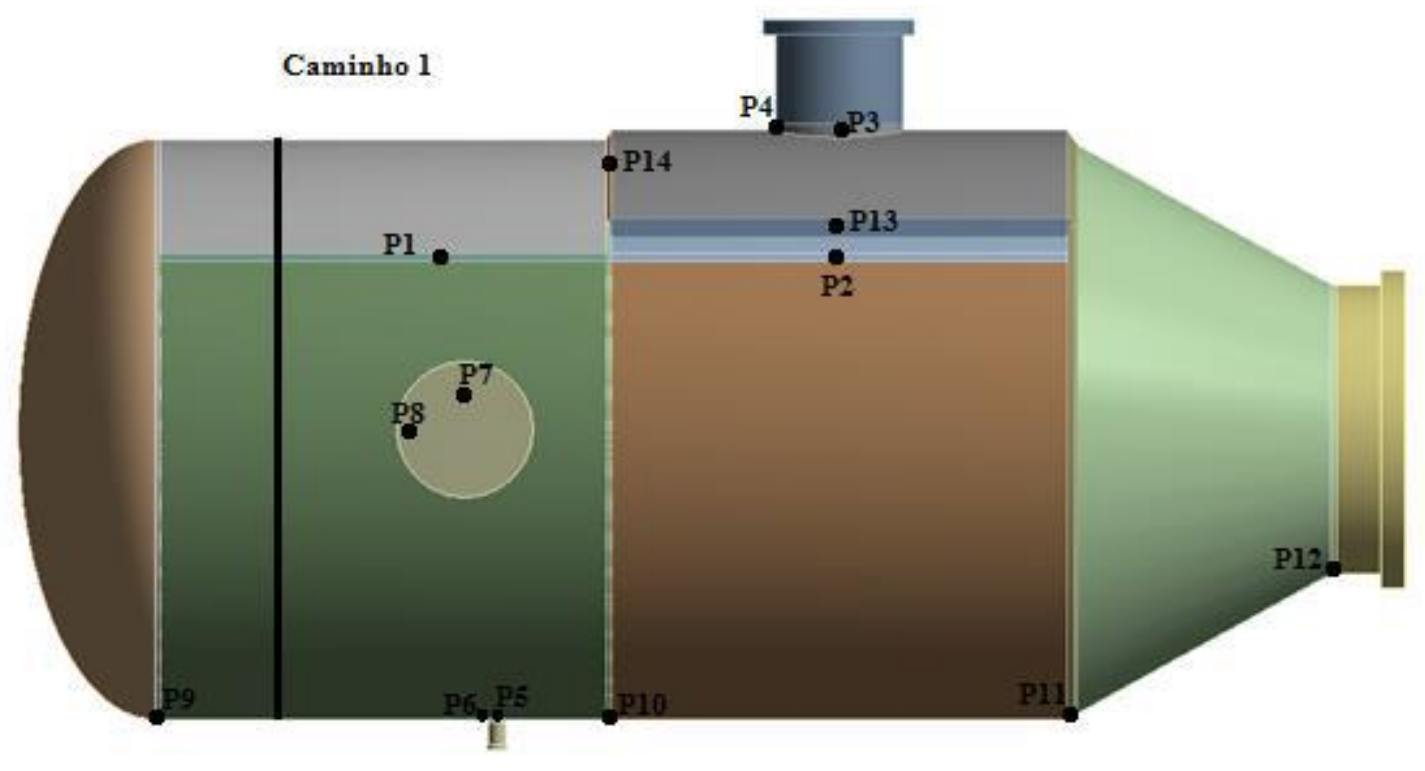

Figura 4.2: Pontos críticos do vaso de pressão [7]

Nas regiões de descontinuidade (as 11 junções), surgem também tensões de flexão, a fim de manter o equilíbrio entre partes diferentes, através da compatibilidade de deformações. Estas tensões de flexão adicionais se superpõem às tensões de membrana (tensões principais causadas pela pressão interna). Além disso, nas regiões das juntas dos bocais, há ainda a concentração de tensões (que multiplicam as tensões trativas).

As tensões nos pontos críticos não são possíveis de serem calculadas analiticamente. Na referência [7], foram obtidas numericamente, por meio do método dos elemento finitos (MEF), as tensões circunferenciais e longitudinais nos 14 pontos da Figura 4.2, cujos valores são mostrados na Tabela 4.2. 


\begin{tabular}{|c|c|c|c|c|}
\hline \multirow{2}{*}{ Ponto } & \multicolumn{2}{|c|}{ Pressão de Projeto } & \multicolumn{2}{c|}{ Pressão de TH } \\
\cline { 2 - 5 } & $\sigma_{\text {Long. }}(\mathbf{M P a})$ & $\boldsymbol{\sigma}_{\text {Circ. }}(\mathbf{M P a})$ & $\boldsymbol{\sigma}_{\text {Long. }}(\mathbf{M P a})$ & $\boldsymbol{\sigma}_{\text {Circ. }}$ (MPa) \\
\hline P1 & 37.00 & 63.84 & 48.13 & 83.03 \\
\hline P2 & 44.17 & 85.79 & 57.42 & 111.59 \\
\hline P3 & -4.77 & 18.11 & -6.23 & 23.55 \\
\hline P4 & 88.16 & 91.59 & 114.67 & 119.13 \\
\hline P5 & 43.17 & 62.21 & 56.11 & 80.90 \\
\hline P6 & 11.71 & 46.04 & 15.26 & 59.90 \\
\hline P7 & -4.22 & 51.92 & -5.37 & 67.41 \\
\hline P8 & 95.24 & 172.65 & 73.20 & 206.18 \\
\hline P9 & 24.97 & -11.51 & 32.48 & -14.98 \\
\hline P10 & 36.88 & 70.68 & 47.95 & 91.91 \\
\hline P11 & -90.71 & -52.88 & -117.96 & -68.77 \\
\hline P12 & 55.31 & 69.37 & 71.94 & 90.22 \\
\hline P13 & 19.83 & 7.45 & 25.78 & 9.71 \\
\hline P14 & -1.21 & 25.74 & -1.57 & 33.47 \\
\hline
\end{tabular}

Tabela 4.2: Tensões longitudinais e circunferenciais dos 14 possíveis pontos críticos [7]

A Pressão de Projeto e de TH de [7] são de 1,441 MPa e 1,874 MPa, respectivamente. Estas pressões são diferentes das deste trabalho (1,3 MPa e 1,845). A referência [7] não considerou corrosão na espessura e, portanto, as espessuras também são diferentes $(12,5 \neq 10,9)$. No entanto, foi identificado que a tanto a pressão quanto a espessura estão relacionadas de forma linear com os valores da Tabela 4.2. Por exemplo, em P1: $\frac{1,874}{1,441} \times$ $37=48,12$. Portanto, usando a Tabela 4.2, podem ser obtidas as tensões longitudinais e circunferenciais para as pressões e espessura deste trabalho.

$\mathrm{Na}$ próxima etapa serão determinados os tipos de detalhes de soldagem das 11 juntas (Figura 1.3). O código de projeto para a análise de fadiga das soldas é o International Institute of Welding (IIW). Para categorizar as juntas dentro das classes de solda do IIW, são assumidas as seguintes hipóteses:

a) As soldas vão de uma face à outra ("atravessam a espessura");

b) Para juntas que podem estar contidas em um intervalo de classes, é adotada uma classe intermediária (e.g., se determinada junta pode ser da Classe 125, 100 ou 80, é adotada a Classe 100). 
Portanto, adotando o modelo do IIW, as Juntas 1, 2, 6, 7, 8, 9, 10 e 11 são do 'tipo 2' - Classe 100 (Tabela 4.4). Já as juntas 3, 4 e 5 são do 'tipo 13' - Classe 80 (Tabela 4.4).

\begin{tabular}{|r|c|c|c|c|}
\multicolumn{1}{|c|}{} & \multicolumn{2}{|c|}{$\mathrm{B}=3.0$} & \multicolumn{2}{c|}{$\mathrm{B}=3.5$} \\
\hline Classe IIW & $\begin{array}{c}\mathrm{C} \\
\left(\mathrm{x10}^{11}\right)\end{array}$ & $\begin{array}{c}\Delta \sigma_{\mathrm{L}}\left(5 \cdot 10^{6}\right) \\
\mathrm{MPa}\end{array}$ & $\begin{array}{c}\mathrm{C} \\
\left(\times 10^{12}\right)\end{array}$ & $\begin{array}{c}\Delta \sigma_{\mathrm{L}}\left(5 \cdot 10^{6}\right) \\
\mathrm{MPa}\end{array}$ \\
\hline 125 & 39.1 & 92 & 43.7 & 96 \\
\hline 112 & 28.1 & 82 & 29.7 & 86 \\
\hline 100 & 20.0 & 74 & 20.0 & 77 \\
\hline 90 & 14.6 & 66 & 13.8 & 69 \\
\hline 80 & 10.2 & 59 & 9.16 & 62 \\
\hline 71 & 7.16 & 52 & 6.03 & 55 \\
\hline 63 & 5.00 & 46 & 3.97 & 48 \\
\hline 56 & 3.51 & 41 & 2.63 & 43 \\
\hline 50 & 2.50 & 37 & 1.77 & 38 \\
\hline 45 & 1.82 & 33 & 1.22 & 35 \\
\hline
\end{tabular}

Tabela 4.3: Constantes das curvas SN normalizadas pelo IIW [12]

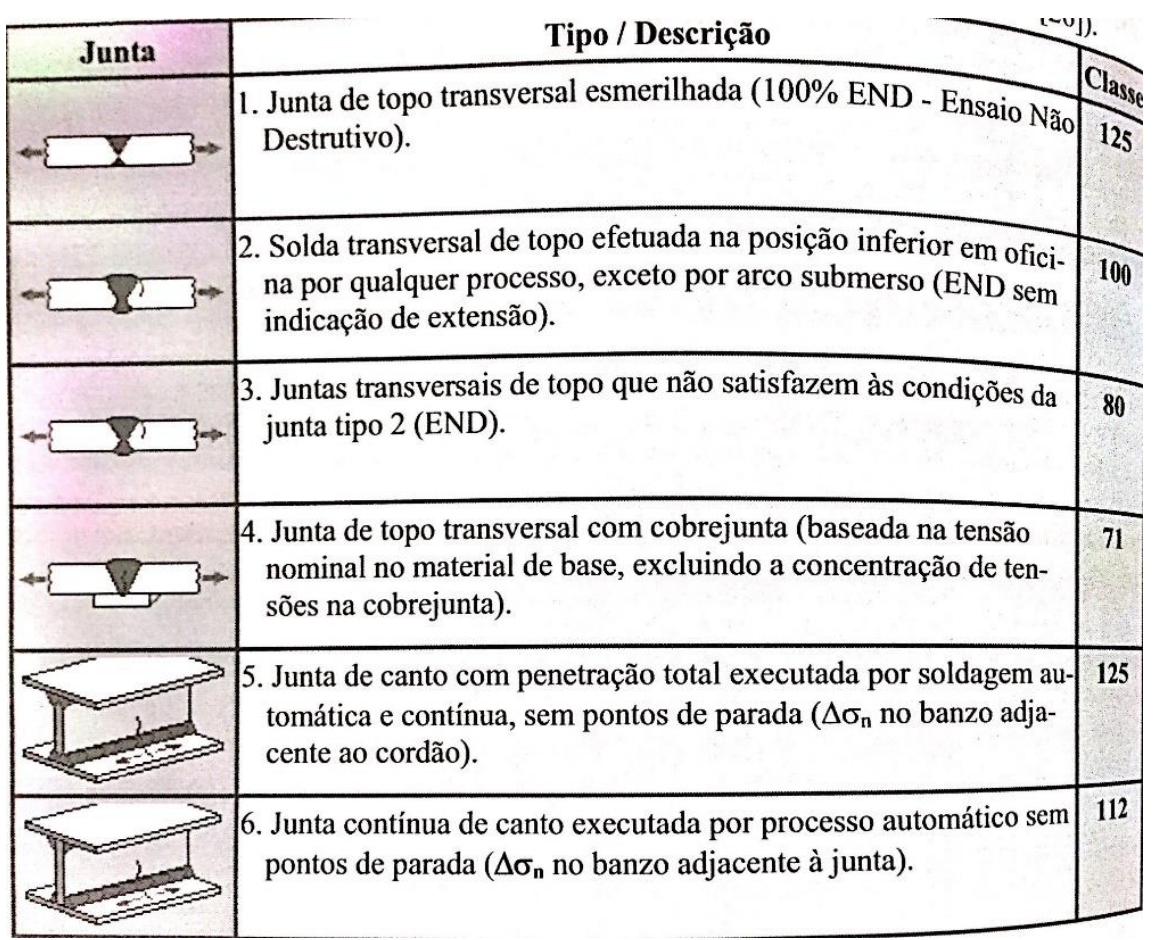

Tabela 4.4: Classes de soldagem normalizadas pelo IIW [12] 


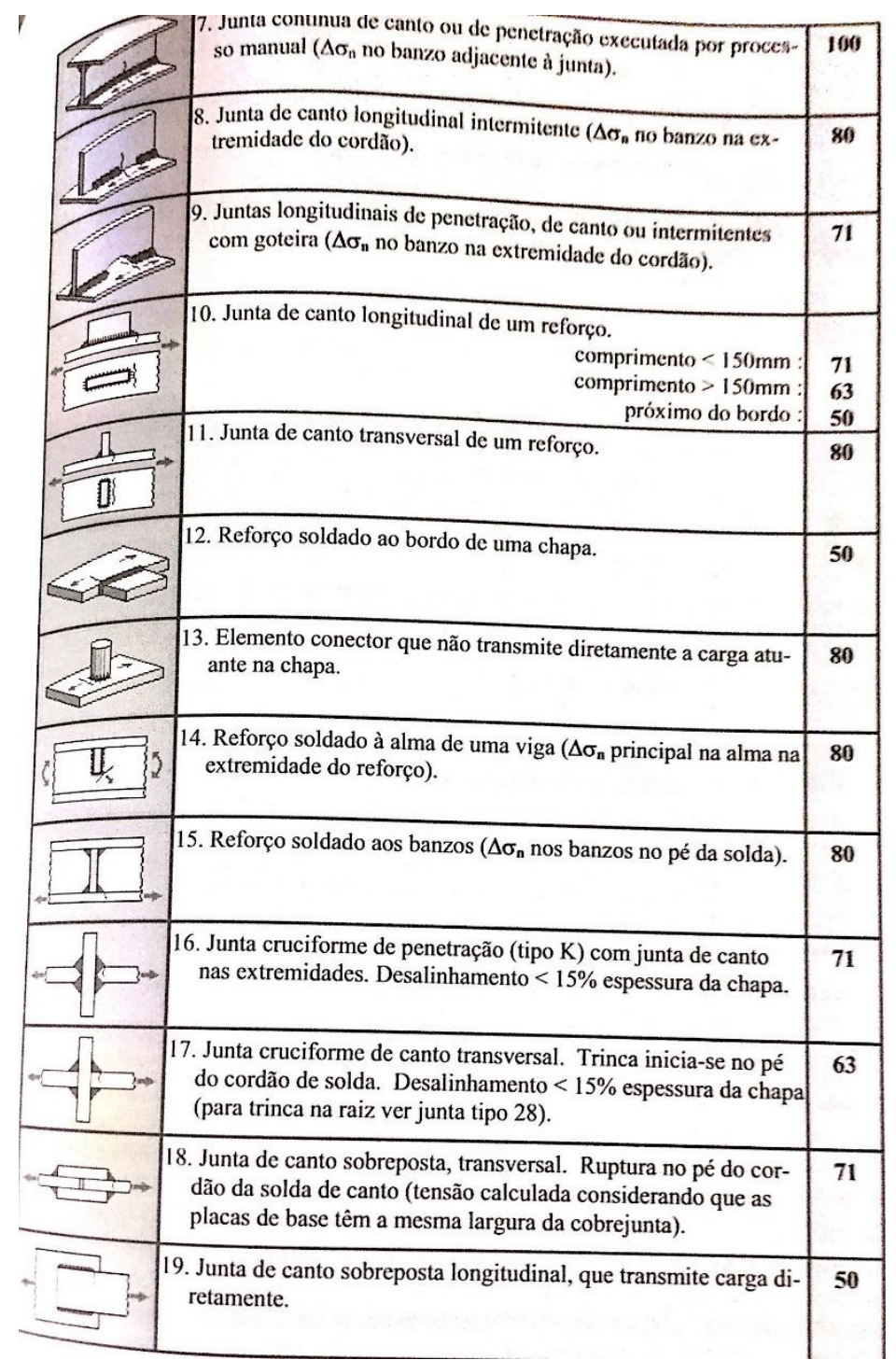

Tabela 4.4 (continuação): Classes de soldagem normalizadas pelo IIW [12]

O fundamento do projeto à fadiga de estruturas soldadas determina que sejam identificados os pontos críticos na estrutura, as gamas das tensões normais que neles atuam e os tipos das juntas soldadas, para, então, calcular as vidas à fadiga usando as curvas SN padronizadas por algum código. As vidas à fadiga de juntas soldadas depende apenas da gama $\Delta \sigma$ das tensões nominais solicitantes e do tipo de junta soldada. As curvas SN são da forma:

$$
\mathrm{N} \Delta \sigma^{\mathrm{B}}=\mathrm{C}
$$

A equação (4-17) usa a gama $\Delta \sigma$ (ao invés de $\sigma_{\mathrm{a}}$ ). 
Aqui, não serão calculadas $\Delta \sigma$ para para todos os 14 pontos críticos. Ao contrário, são determinadas as gamas das tensões nominais de apenas dois pontos para cada uma das classes de solda. Como o intuito é de obter a menor vida dentre os pontos críticos, basta que sejam calculadas as vidas do pontos onde atuam as maiores tensões. Para a Classe 100, estes pontos são P2 e P11; já para a Classe 80, estes pontos são o P8 e o P4. Antes de calcular $\Delta \sigma$ para estes quatro pontos, suas tensões principais devem ser recalculadas de acordo com as pressões e espessura deste trabalho.

Para re-calcular determinada tensão da Tabela 4.2, basta multiplicála pela razão $\frac{\mathrm{P}}{\mathrm{P}^{\prime}}$ e em seguida por $\frac{\mathrm{t}}{\mathrm{t}_{\mathrm{c}}}$, sendo $\mathrm{P}$ a pressão deste trabalho e $\mathrm{P}^{`} \mathrm{a}$ pressão de [7]; enquanto t é a espessura integral e $t_{c}$ a espessura já tendo sofrido corrosão.

\section{P.2:}

$\sigma_{\mathrm{C}_{\mathrm{Op}}}=85,79 \times \frac{1,3}{1,441} \times \frac{12,5}{10,9}=88,8 \mathrm{MPa}$ e $\sigma_{\mathrm{r}_{\mathrm{Op}}}=-1,3 \mathrm{MPa}$

$\sigma_{\mathrm{T}_{\mathrm{Op}}}=\sigma_{1}-\sigma_{3}=88,8-(-1,3)=90,1 \mathrm{MPa}$

Onde $\sigma_{\mathrm{C}_{\mathrm{Op}}}$ denota tensão circunferencial para a pressão de operação; $\sigma_{\mathrm{r}_{\mathrm{Op}}}$ denota tensão radial para a pressão de operação; e $\sigma_{\mathrm{T}_{\mathrm{Op}}}$ denota tensão de Tresca para a pressão de operação.

Para obter as tensões correspondentes ao teste hidrostático, basta multiplicar as tensões associadas à pressão de operação pela razão $\frac{\mathrm{P}_{\mathrm{TH}}}{\mathrm{P}_{\mathrm{Op}}}$, onde $\mathrm{P}_{\mathrm{TH}}$ é a pressão do teste hidrostático e $\mathrm{P}_{\mathrm{Op}}$ é a pressão de operação. Esta razão é constante e igual a 1,85/1,3 $=1,42$.

$$
\begin{aligned}
& \sigma_{\mathrm{C}_{\mathrm{TH}}}=88,8 \times 1,42=126 \mathrm{MPa} \text { e } \sigma_{\mathrm{r}_{\mathrm{TH}}}=-1,85 \mathrm{MPa} \\
& \sigma_{\mathrm{T}_{\mathrm{TH}}}=\sigma_{1}-\sigma_{3}=126-(-1,85) \cong 128 \mathrm{MPa}
\end{aligned}
$$

Onde, novamente, os primeiros indices das tensões ( $\mathrm{C}, \mathrm{r}$ e $\mathrm{T}$ ) significam circunferencial, normal (ou radial) e Tresca, respectivamente.

Finalmente, para obter as tensões correspondentes à pressão atmosférica, basta multiplicar as tensões associadas à pressão de operação 
pela razão $\frac{\mathrm{P}_{\mathrm{atm}}}{\mathrm{P}_{\mathrm{Op}}}$, onde $\mathrm{P}_{\mathrm{atm}}$ é a pressão atmosférica e $\mathrm{P}_{\mathrm{Op}}$ é a pressão de operação. Esta razão é constante e igual a 0,101/1,3 = 0,0777

$$
\begin{aligned}
& { }^{\sigma} \mathrm{C}_{\mathrm{amb}}=88,8 \times 0,0777=6,9 \mathrm{MPa} \text { e } \sigma_{\mathrm{r}_{\mathrm{amb}}}=-0,101 \mathrm{MPa} \\
& { }^{\sigma} \mathrm{T}_{\mathrm{amb}}=\sigma_{1}-\sigma_{3}=6,9-(-0,101) \cong 7 \mathrm{MPa}
\end{aligned}
$$

Onde o sub índice 'amb' significa ambiente.

Com os máximos e mínimos dos ciclos de operação e dos ciclos de $\mathrm{TH}$, podem ser obtidas as gamas de tensão associadas. Lembrando que os ciclos de operação são aqueles associados com uma variação de pressão $[0,101 \rightarrow 1,3 \rightarrow 0,101]$ e os ciclos de TH são aqueles cuja correspondente variação de pressão é $[0,101 \rightarrow 1,85 \rightarrow 0,101]$.

$$
\begin{aligned}
& \Delta \sigma_{\mathrm{TH}}=128-7=121 \mathrm{MPa} \\
& \Delta \sigma_{\mathrm{Op}}=90-7=83 \mathrm{MPa}
\end{aligned}
$$

\section{P.11:}

$$
\begin{aligned}
& \sigma_{1_{O p}}=\sigma_{\mathrm{r}_{\mathrm{Op}}}=-1,3 \mathrm{MPa} \text { e } \sigma_{3_{O p}}=\sigma_{\mathrm{l}_{\mathrm{Op}}}=-93,8 \mathrm{MPa} \\
& \sigma_{\mathrm{T}_{\mathrm{Op}}}=\sigma_{1_{O p}}-\sigma_{3_{O p}}=-1,3-(-93,8)=92,5 \mathrm{MPa} \\
& \sigma_{1_{T H}}=\sigma_{\mathrm{r}_{\mathrm{TH}}}=-1,85 \mathrm{MPa} \text { e } \sigma_{3_{T H}}=\sigma_{\mathrm{l}_{\mathrm{TH}}}=-134 \mathrm{MPa} \\
& \sigma_{\mathrm{T}_{\mathrm{TH}}}=\sigma_{1_{T H}}-\sigma_{3_{T H}}=-1,85-(-134) \cong 132 \mathrm{MPa} \\
& \sigma_{1_{a m b}}=-0,101 \mathrm{MPa} \text { e } \sigma_{3_{a m b}}=-7,3 \mathrm{MPa} \\
& \sigma_{\mathrm{T}_{\mathrm{amb}}}=\sigma_{1_{a m b}}-\sigma_{3_{a m b}}=-0,101-(-7,3) \cong 7,2 \mathrm{MPa}
\end{aligned}
$$

Onde o sub índice 'amb' significa ambiente (temperatura de $25 \mathrm{C}$ e $\mathrm{P}=$ Patm).

$$
\begin{gathered}
\Delta \sigma_{\mathrm{TH}}=132-7,2=125 \mathrm{MPa} \\
\Delta \sigma_{\mathrm{Op}}=92,5-7,2=85,3 \mathrm{MPa}
\end{gathered}
$$




\section{P.4:}

$$
\begin{aligned}
& \sigma_{1_{o p}}=\sigma_{\mathrm{C}_{\mathrm{Op}}}=94,8 \mathrm{MPa} \text { e } \sigma_{3_{O p}}=\sigma_{\mathrm{r}_{\mathrm{Op}}}=-1,3 \mathrm{MPa} \\
& \sigma_{\mathrm{T}_{\mathrm{Op}}}=\sigma_{1_{O p}}-\sigma_{3_{O p}}=94,8-(-1,3)=96,1 \mathrm{MPa} \\
& \sigma_{1_{T H}}=\sigma_{\mathrm{C}_{\mathrm{TH}}}=135 \mathrm{MPa} \text { e } \sigma_{3_{T H}}=\sigma_{\mathrm{r}_{\mathrm{TH}}}=-1,85 \mathrm{MPa} \\
& \sigma_{\mathrm{T}_{\mathrm{TH}}}=\sigma_{1_{T H}}-\sigma_{3_{T H}}=135-(-1,85) \cong 137 \mathrm{MPa} \\
& \sigma_{1_{a m b}}=7,4 \mathrm{MPa} \text { e } \sigma_{3_{a m b}}=-0,101 \mathrm{MPa} \\
& \sigma_{\mathrm{T}_{\mathrm{amb}}}=\sigma_{1_{a m b}}-\sigma_{3_{a m b}}=7,4-(-0,101) \cong 7,5 \mathrm{MPa} \\
& \Delta \sigma_{\mathrm{TH}}=137-7,5=130 \mathrm{MPa} \\
& \Delta \sigma_{\mathrm{Op}}=96,1-7,5=88,6 \mathrm{MPa}
\end{aligned}
$$

\section{P.8:}

$$
\begin{aligned}
& \sigma_{1_{o p}}=\sigma_{\mathrm{C}_{\mathrm{Op}}}=179 \mathrm{MPa} \text { e } \sigma_{3_{o p}}=\sigma_{\mathrm{r}_{\mathrm{Op}}}=-1,3 \mathrm{MPa} \\
& \sigma_{\mathrm{T}_{\mathrm{Op}}}=\sigma_{1_{\rho_{0}}}-\sigma_{3_{O p}}=179-(-1,3)=180 \mathrm{MPa} \\
& \sigma_{1_{T H}}=\sigma_{\mathrm{C}_{\mathrm{TH}}}=245 \mathrm{MPa} \text { e } \sigma_{3_{T H}}=\sigma_{\mathrm{r}_{\mathrm{TH}}}=-1,85 \mathrm{MPa} \\
& \sigma_{\mathrm{T}_{\mathrm{TH}}}=\sigma_{1_{T H}}-\sigma_{3_{T H}}=245-(-1,85) \cong 247 \mathrm{MPa} \\
& \sigma_{1_{a m b}}=13,9 \mathrm{MPa} \text { e } \sigma_{3_{a m b}}=-0,101 \mathrm{MPa} \\
& \sigma_{\mathrm{T}_{\mathrm{amb}}}=\sigma_{1_{a m b}}-\sigma_{3_{a m b}}=13,9-(-0,101) \cong 14 \mathrm{MPa} \\
& \Delta \sigma_{\mathrm{TH}}=247-14=233 \mathrm{MPa} \\
& \Delta \sigma_{\mathrm{Op}}=180-14=166 \mathrm{MPa}
\end{aligned}
$$

Todas as tensões, com exceção da tensão circunferencial do TH em P.8 (e também da tensão de Tresca associada), estão abaixo das tensões de escoamento. Por haver escoamento em P.8, há alguma imprecisão na linearidade assumida para este ponto, e consequentemente no modelo SN. No entanto, a tensão de Tresca deste ponto ultrapassa a tensão de 
escoamento em somente $12 \%$. E, o que é mais importante, há somente cinco TH. Desta forma, o modelo SN não irá gerar previsões ruins.

Os pontos P.2 e P.11 estão localizados nas juntas 2 e 8, respectivamente, pertencendo à Classe 100 de solda. Já os pontos P.4 e P.8 ficam nas juntas 3 e 5, que são da Classe 80.

Da equação (4-17): $N=\frac{C}{\Delta \sigma^{B}}$

Para B $=3,0$ e Classe 100, C $=2 \times 10^{12}$ (Tabela 4.3).

$\mathrm{E}$, para $\mathrm{B}=3,0$ e Classe $80, \mathrm{C}=10,2 \times 10^{11}$ (Tabela 4.3).

P.2

$$
\begin{aligned}
& \mathrm{N}_{\mathrm{TH}}=\frac{\mathrm{C}}{\Delta \sigma_{\mathrm{TH}}{ }^{\mathrm{B}}}=\frac{2 \times 10^{12}}{121^{3}}=1,12 \times 10^{6} \text { ciclos } \\
& \therefore \mathrm{D}_{\mathrm{TH}}=\frac{\mathrm{n}_{\mathrm{TH}}}{\mathrm{N}_{\mathrm{TH}}}=\frac{5}{1,12 \times 10^{6}}=4,43 \times 10^{-6} \cong 0 \\
& \therefore \mathrm{n}_{\mathrm{Op}}=\mathrm{N}_{\mathrm{Op}}=\frac{2 \times 10^{12}}{83^{3}}=3,5 \times 10^{6} \text { ciclos }
\end{aligned}
$$

\section{P.11}

$$
\begin{aligned}
& \mathrm{N}_{\mathrm{TH}}=\frac{2 \times 10^{12}}{125^{3}}=1,024 \times 10^{6} \text { ciclos } \\
& \therefore \mathrm{D}_{\mathrm{TH}}=\frac{\mathrm{n}_{\mathrm{TH}}}{\mathrm{N}_{\mathrm{TH}}}=\frac{5}{1,024 \times 10^{6}}=4,88 \times 10^{-6} \cong 0 \\
& \therefore \mathrm{n}_{\mathrm{Op}}=\mathrm{N}_{\mathrm{Op}}=\frac{2 \times 10^{12}}{85,3^{3}}=3,22 \times 10^{6} \text { ciclos }
\end{aligned}
$$

\section{P.4}

$$
\begin{gathered}
\mathrm{N}_{\mathrm{TH}}=\frac{\mathrm{C}}{\Delta \sigma_{\mathrm{TH}}{ }^{\mathrm{B}}}=\frac{10,2 \times 10^{11}}{130^{3}}=4,64 \times 10^{5} \text { ciclos } \\
\therefore \mathrm{D}_{\mathrm{TH}}=\frac{5}{4,64 \times 10^{5}}=1,07 \times 10^{-5} \cong 0 \\
\therefore \mathrm{n}_{\mathrm{Op}}=\mathrm{N}_{\mathrm{Op}}=\frac{10,2 \times 10^{11}}{88,6^{3}}=1,46 \times 10^{6} \text { ciclos }
\end{gathered}
$$




\section{P.8}

$$
\begin{array}{r}
\mathrm{N}_{\mathrm{TH}}=\frac{\mathrm{C}}{\Delta \sigma_{\mathrm{TH}}{ }^{\mathrm{B}}}=\frac{10,2 \times 10^{11}}{233^{3}}=80636 \text { ciclos } \\
\therefore \mathrm{D}_{\mathrm{TH}}=\frac{5}{80636}=6,2 \times 10^{-5} \cong 0 \\
\therefore \mathrm{n}_{\mathrm{Op}}=\mathrm{N}_{\mathrm{Op}}=\frac{10,2 \times 10^{11}}{166^{3}}=222000 \text { ciclos }
\end{array}
$$

Se for usado um fator de segurança contra fadiga, por exemplo $\varphi_{F}=$ 1,5 , os resultados serão:

$$
\begin{aligned}
& \mathrm{N}_{\mathrm{TH}}=\frac{\mathrm{C}}{\left(1,5 \times \Delta \sigma_{\mathrm{TH}}\right)^{\mathrm{B}}}=\frac{10,2 \times 10^{11}}{1,5^{3} \times 233^{3}}=23892 \text { ciclos } \\
& \therefore \mathrm{D}_{\mathrm{TH}}=\frac{5}{23892}=2,09 \times 10^{-4} \\
& \mathrm{~N}_{\mathrm{Op}}=\frac{10,2 \times 10^{11}}{1,5^{3} \times 166^{3}}=66069 \\
& \mathrm{n}_{\mathrm{Op}}=\mathrm{N}_{\mathrm{Op}}\left(1-\mathrm{D}_{\mathrm{TH}}\right)=66069 \times 0,9998=66055
\end{aligned}
$$

\begin{tabular}{|c|c|}
\hline \multicolumn{2}{|c|}{ Vidas à Fadiga nos Pontos Críticos } \\
\hline Ponto & Vida ( $n^{\circ}$ de ciclos $)$ \\
\hline P. 2 & $3,5 \times 10^{6}$ \\
\hline P.11 & $3,22 \times 10^{6}$ \\
\hline P.4 & $1,46 \times 10^{6}$ \\
\hline P.8 & $\begin{array}{l}222000\left(\operatorname{sem} \varphi_{F}\right) \mathrm{e} \\
66055\left(\operatorname{com} \varphi_{F}\right)\end{array}$ \\
\hline
\end{tabular}

ciclos

A Tabela 4.5 apresenta as vidas encontradas nos pontos críticos:

Tabela 4.5: Vidas à fadiga nos pontos críticos

O Ponto 8 é o que tem a menor vida. Portanto, o número de ciclos de operação que P. 8 pode suportar define a vida deste vaso de pressão, sendo este valor 222000 ciclos sem fator de segurança e 66055 com um fator de segurança contra fadiga de 1,5. Estas vidas são inferiores ao limite de fadiga definido pela IIW $\left(5 \times 10^{6}\right.$ ciclos $)$, assim como ao limite de fadiga 
para aços (mínimo de $10^{6}$ ciclos). No entanto, 222000 é uma vida longa e 66055 é muito maior do que 1000 (o limite inferior do número de ciclos, para aços, no Método SN), o que mostra que o Método SN foi bem usado.

\section{2}

\section{Avaliação da fadiga segundo a norma ASME}

A norma ASME trata de fadiga em vasos de pressão no item 5.5 da Divisão 2, Seção VIII [2].

Segundo a norma (e também segundo a teoria), uma análise de fadiga deve ser feita em um componente que esteja sujeito a carregamentos cíclicos. A avaliação de fadiga é feita com base no número de ciclos de gamas de tensão ou deformação que são aplicados em um ponto do componente [2].

A norma estabelece meios de se verificar se a análise detalhada de fadiga é necessária. Estes meios, apresentados no parágrafo 5.5.2 do ASME, são discutidos a seguir.

São fornecidas três opções que, se satisfeitas, dispensam os cálculos de fadiga no projeto. A opção de se ter experiência prévia com equipamento similar operando sob condições semelhantes ( $1^{\text {a }}$ opção) não é satisfeita no caso aqui tratado. Como a tensão de ruptura do material é inferior a $552 \mathrm{MPa}$ (Tabela 2.1), devem ser seguidas as regras de checagem pelo Método A [2]. Não serão transcritos aqui todos os passos, mas é facil checar que foram cumpridos:

1) Passo 1: História de tensões determinada.

2) Passo 2: $\mathrm{N}_{\Delta \mathrm{FP}_{\mathrm{Op}}}=222000$ e $\mathrm{N}_{\Delta \mathrm{FP}_{\mathrm{TH}}}=5$

3) Passo 3: $N_{\triangle P O}=\frac{(1,85-0,101)}{1,527}=1,15<120 \%$ (ou $20 \%$ de acréscimo) $\therefore N_{\triangle P O}=0$ 
4) Passo 4: Embora haja ciclos térmicos, devido ao gradiente de temperatura ser baixo (4.1.2), a diferença de temperaturas entre a superfície interna e externa nunca será superior aos $28{ }^{\circ} \mathrm{C}$. Portanto, o fator multiplicativo dos ciclos térmicos (Tabela 5.8 de [2]) será zero, o que acarreta em $N_{\Delta T E}=0$.

5) Passo 5: Como as placas e componentes do vaso têm um mesmo coeficiente de expansão térmica (pois são do mesmo material), $N_{\Delta T \alpha}=0$.

6) Segundo a Tabela 5.9 de [2], a condição para que a avaliação de fadiga seja dispensada é: $N_{\triangle F P}+N_{\triangle P O}+N_{\Delta T E}+N_{\Delta T \alpha} \leq 1000$

$$
222005+0+0+0 \gg 1000
$$

Portanto, a fadiga deve ser levada em conta no projeto ou análise do vaso de pressão.

É importante destacar que, nos passos 1 e 2, como não é conhecido a priori o número de ciclos de operação que será aplicado, foi usada a vida encontrada pela teoria (Subseção 4.1.4) para simular este número. Esta vida é na verdade o número máximo de ciclos que poderiam ser aplicados. 


\section{5}

\section{CONCLUSÃO E SUGESTÕES PARA TRABALHOS FUTUROS}

A MPAT e a PTH são umas das primeiras coisas que se calculam em vasos de pressão. No entanto, não são suficientes por si só para garantir a integridade estrutural. Podem por exemplo existir carregamentos externos, que não têm nenhuma relação com a pressão interna. Ou então, conforme foi visto, podem existir tensões cíclicas na estrutura, que surgem por causa das condições de operação, fazendo-se também necessária uma análise de fadiga no vaso de pressão.

A vida à fadiga deve ser sempre avaliada nos pontos críticos, pois é onde se iniciam as trincas. Este vaso de pressão, para estas condições de operação, apresenta uma vida bastante alta (apesar de não ser infinita), mesmo com fator de segurança. Geralmente vasos de pressão não são submetidos a esta quantidade toda de ciclos.

Por fim, deve-se salientar, que um projeto e análise completos de vasos de pressão reais vão muito além do que foi abordado neste trabalho.

Para trabalhos futuros, é sugerido fazer o dimensionamento dos suportes; supor e levar em conta na análise estrutural cargas externas que possam existir; supor condições de operação que variem e fazer os cálculos teóricos de fadiga utilizando o Critério de Mises; fazer uma análise de fadiga na estrutura do vaso também através da norma ASME. 


\section{REFERÊNCIAS BIBLIOGRÁFICAS:}

[1] ASME BPVC, Section VIII, Division 1, 2010

[2] ASME BPVC, Section VIII, Division 2, 2010

[3] ASME BPVC, Section II, Part D, 2010

[4] Megyesy, Eugene F. Pressure Vessel Handbook, 12 ed., 2001

[5] Moss, D. Pressure Vessel Design Manual, 3 ed., 2004

[6] Chattopadhyay, S. Pressure Vessels Design and Practice, CRC Press, 2005

[7] Paiva, Vitor E. L. Simulação de tensões residuais de soldagem e seu alívio por teste hidrostático, Dissertação de Mestrado, PUC - Rio, 2015

[8] Da Silva JR, V. F. Projeto Estrutural e de Fabricação de Vaso de Pressão Esférico, T.C.C., USP, 2011

[9] Iecker, T. D. Análise de Tensões em Vasos de Pressão Através do Método de Elementos Finitos, CEFET/RJ, 2014

[10] http://www.nacionaltampos.com.br/index.php?link=tamposindustriais\#tampos

[11] Van Wylen, Fundamentos da Termodinâmica, $7^{\mathrm{a}}$ ed., Blucher, 2010 Apêndice B

[12] Pinho de Castro, J. T.; Meggiolaro, M. A. Fadiga - Técnicas e Práticas de Dimensionamento Estrutural sob Cargas Reais de Serviço, 2009

[13] Gere, J. M.; Goodno, B. J. Mecânica dos Materiais, $7^{a}$ ed., Cengage Learning 


\section{ANEXO A - CÁLCULO DA CAPACIDADE VOLUMÉTRICA}

A capacidade volumétrica do vaso pode ser encontrada somando-se os volumes de um cilindro; de um tronco de cone e de um tampo elipsoidal (ou torisférico). Evidentemente devem ser usadas as dimensões internas.

Para um cilindro de raio $R$ e altura $H, V=\pi R^{2} H$. Assim, $V=3,1416 \times$ $0,625^{2} \times 2=2,454 \mathrm{~m}^{3}$ ou $2454 \mathrm{~L}$

Para um tronco de cone de diâmetro maior D; diâmetro menor d e altura $\mathrm{h}$, $\mathrm{V}=0,2618 \mathrm{~h}\left(\mathrm{D}^{2}+\mathrm{Dd}+\mathrm{d}^{2}\right)$. Desta forma, $\mathrm{V}=0,2618 \times 0,58\left(1,25^{2}+\right.$ $\left.1,25 \times 0,625+0,625^{2}\right)=0,415 \mathrm{~m}^{3}$ ou $415 \mathrm{~L}$

Finalmente, de acordo com [nacionaltampos] para um tampo torisférico ASME de diâmetro interno $\mathrm{D}, \mathrm{V}=0,08 \mathrm{D}^{3}$. Assim, $\mathrm{V}=0,08 \times 1,25^{3}=$ $0,156 \mathrm{~m}^{3}$ ou $156 \mathrm{~L}$.

Portanto, o volume total é de: $2,454+0,415+0,156=3,025 \mathrm{~m}^{3}$ ou $3025 \mathrm{~L}$.

\section{ANEXO B - TENSÕES PRINCIPAIS EM VASOS CILÍNDRICOS}

Seja um tanque circular de parede fina $\mathrm{AB}$, submetido a uma pressão interna p (Figura B-1 (a)). Está representado um elemento de tensão na parede do tanque, cujas tensões normais são $\sigma_{1}$ e $\sigma_{2}$, que também são as tensões principais.

Para obter a tensão principal $\sigma_{1}$, também chamada de tensão circunferencial, são feitos dois cortes transversais ( $\mathrm{mn}$ e pq) ao eixo longitudinal do tanque AB (Figura B-1 (a)), sendo então obtido o corpo livre mostrado na Figura B-1 (b). Do equilíbrio deste corpo livre:

$$
\begin{aligned}
& \sigma_{1} 2 \mathrm{bt}-\mathrm{pb} 2 \mathrm{r}=0 \therefore \sigma_{1} \mathrm{bt}=\mathrm{pbr} \\
& \sigma_{1}=\frac{\mathrm{pr}}{\mathrm{t}}
\end{aligned}
$$

Onde $\mathrm{b}$ é a distância entre os planos mn e pq, t é a espessura da parede, $\mathrm{r}$ é o raio interno e p é a pressão interna. 
A tensão principal $\sigma_{2}$, também chamada de tensão longitudinal, pode ser obtida de forma semelhante ao que foi feito anteriormente. Fazendo-se desta vez somente o corte $\mathrm{mn}$ no tanque $\mathrm{AB}$, é obtido o corpo livre mostrado na Figura B-1 (c). Do equilíbrio deste corpo:

$$
\begin{aligned}
& \sigma_{2} 2 \pi r t-p \pi r^{2}=0 \therefore \sigma_{2} 2 t=p r \\
& \sigma_{2}=\frac{p r}{2 t}=\frac{\sigma_{1}}{2}
\end{aligned}
$$



(a)

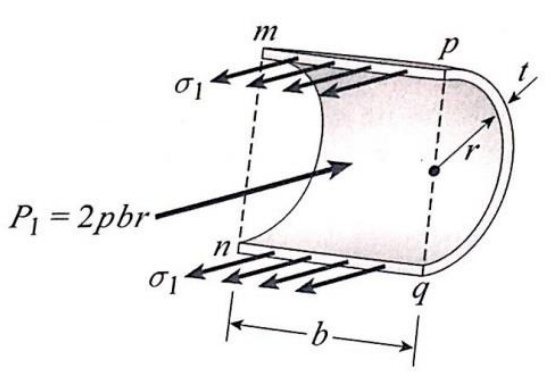

(b)

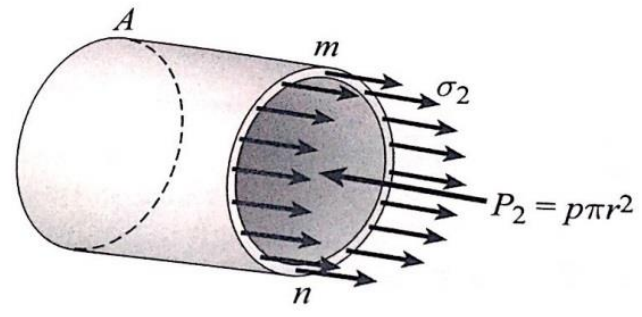

(c)

Figura B-1: Tanque AB e as suas seções (a), (b) e (c) [13]

Em vasos de pressão de parede fina $(\mathrm{r} / \mathrm{t}>10), \sigma_{1}$ e $\sigma_{2}$ agem uniformemente ao longo da espessura. Há uma terceira tensão principal, $\sigma_{3}$, que age na direção radial. Assim, para vasos de pressão sujeitos à pressões internas $\mathrm{p}_{\mathrm{i}}, \sigma_{3}=-\mathrm{p}_{\mathrm{i}}$ e agirá somente na superfície interna do casco. Enquanto que em vasos de pressão sujeitos à pressões externas $\mathrm{p}_{\mathrm{e}}$, $\sigma_{3}=-p_{e}$ e agirá somente na superfície externa do casco.

As tensões $\sigma_{1}$ e $\sigma_{2}$ são chamadas de tensões de membrana, enquanto que $\sigma_{3}$ é a tensão radial (ou normal). Em vasos de pressão de parede fina, sempre $\sigma_{\mathrm{C}}>\sigma_{\mathrm{l}}>\sigma_{\mathrm{r}}$, daí o motivo de designá-las (da esquerda para direita) por $\sigma_{1}, \sigma_{2}$ e $\sigma_{3}$. 
Para se projetar um vaso de pressão de paredes finas contra o escoamento e usando o critério de Tresca, basta fazer:

$$
\begin{gathered}
\sigma_{\mathrm{T}}=2 \tau_{\text {máx }}=\sigma_{1}-\sigma_{3}=\mathrm{S}_{\mathrm{E}} \\
\frac{\mathrm{pr}}{\mathrm{t}}-\mathrm{p}=\mathrm{S}_{\mathrm{E}} \\
\therefore \mathrm{p}\left(\frac{\mathrm{r}}{\mathrm{t}}+1\right)=\frac{\mathrm{S}_{\mathrm{E}}}{\varphi_{\mathrm{E}}}
\end{gathered}
$$

Onde $\tau_{\text {máx }}$ é a máxima tensão cisalhante e $\varphi_{\mathrm{E}}$ é um fator de segurança conta o escoamento, que depende do material e do uso do vaso.

$r$ é o raio médio do vaso. No entanto, para estar do lado da segurança, pode-se superestimar $r$ pelo raio externo do vaso. 